\title{
Hybrid Approach in Microscale Transport Phenomena: Application to Biodiesel Synthesis in Microreactors
}

\author{
J.M. Costa Jr. ${ }^{1}$, P.C. Pontes ${ }^{2,3}$, C.P. Naveira-Cotta ${ }^{3,4}$, \\ M.K. Tiwari ${ }^{3}$, S. Balabani ${ }^{5}$, R.M. Cotta ${ }^{4,5,6^{*}}$ \\ ${ }^{1}$ Instituto Federal Sertão Pernambucano, IFSERTÃO-PE, Serra Talhada, PE, Brazil \\ ${ }^{2}$ Univ. Federal do Sul e Sudeste do Pará, UNIFESSPA, Santana do Araguaia, PA, Brazil \\ ${ }^{3}$ Nanoengineered Systems Laboratory, UCL Mechanical Engineering, University College \\ London, London, UK, WC1E 7JE \\ ${ }^{4}$ Laboratory of Nano and Microfluidics and Microsystems, Mechanical Engineering and \\ Nanoengineering Depts., POLI \& COPPE, Universidade Federal do Rio de Janeiro, Brazil. \\ ${ }^{5}$ UCL Mechanical Engineering, University College London, London, UK, WC1E 7JE \\ ${ }^{6}$ Interdisciplinary Nucleus for Social Development, NIDES/CT, UFRJ, Brazil. \\ "Corresponding Author: cotta@mecanica.coppe.ufrj.br
}

\begin{abstract}
A hybrid engineering approach to the study of transport phenomena, based on the synergy among computational, analytical, and experimental methodologies is reviewed. The focus of the chapter is on fundamental analysis and proof of concept developments in the use of nano- and micro-technologies for energy efficiency and heat and mass transfer enhancement applications. The hybrid approach described herein combines improved lumped-differential modeling, hybrid numericalanalytical solution methods, mixed symbolic-numerical computations, and advanced experimental techniques for micro-scale transport phenomena. An application dealing with micro-reactors for continuous synthesis of biodiesel is selected to demonstrate the instrumental role of the hybrid approach in achieving improved design and enhanced performance.
\end{abstract}

Keywords: Hybrid methods, micro-scale transport phenomena, integral transforms, inverse problems, lumped models, biodiesel synthesis, micro-reactors 


\section{INTRODUCTION}

Global demands for water, energy and food are estimated to increase by $40 \%, 50 \%$ and $35 \%$, respectively, by 2030 [1], mainly due to global population increase and growth of middle class and its associated consumption pattern, with the inherent consequences on climate change and environmental impact as a whole. Thus, the development of increasingly more sustainable processes is a driving force on most of the recent research efforts in engineering [2], including transport phenomenabased processes where the design of improved heat and mass exchange equipment is an essential technological goal [3]. For instance, over the last few decades, heat exchangers employing micro- and mini-channels have been progressively preferred in applications that require high heat removal demands and/or limited dimensions [3-7].

The research reported here originated through a UK-Brazil Newton FundFAPERJ collaborative development project, offering a holistic approach to address the complex nexus challenges from the societal demand for food, water, energy and need for environmental care. The motivation involved a combined system, harnessing electrical energy from high efficiency water cooled HCPVs and using the resulting waste heat for water desalination and continuous biodiesel production, making use of nano and micro-scale transport phenomena analysis tools recently employed in different thermal engineering applications [8-11]. The original aspects of the present research includes problem formulation and simplification strategies, modern analytical-numerical solution methods for differential equations, parametric and functional identification through inverse analysis, symbolic computation tools, nano and micro-fabrication, non-intrusive optical techniques in micro-scale heat and fluid flow, employed individually or jointly, as needed to answer the basic questions raised by the applications to be advanced. This chapter starts by describing the so-called hybrid approach that combines the computational-analytical-experimental tools to analyze a system and its behavior [12-13]. The impact of nano- and microtechnologies on transport enhancement has already been illustrated through some fundamental analysis around the three major paths [8], namely, miniaturization of heat exchange devices, thus bringing transport characteristic dimensions to the micro-scale, functionalization of the materials (solids and fluids) at the nanoscale, thus augmenting thermophysical properties, and geometrical structuring of micro-systems and passages, thus altering the transport or mixing mechanisms. These fundamental ideas and the hybrid analysis framework are here exemplified in a selected application involving the continuous synthesis of biodiesel in micro-reactors.

\section{THE HYBRID APPROACH}

Figure 1 depicts the various steps in simulating a typical advanced engineering application [12-13], starting with the construction of a physical model of the (usually intricate) real problem. Quite frequently, working on nano- and micro- 
technologies implementation, requires the problems to be modeled within a multiphysics and multiscale framework. The various stages depicted in Fig. 1 are interconnected; hence, in most simulation processes parallel improvements in each stage are necessary, in order to achieve a desirable combination of accuracy improvement and simulation cost reduction in the overall development.

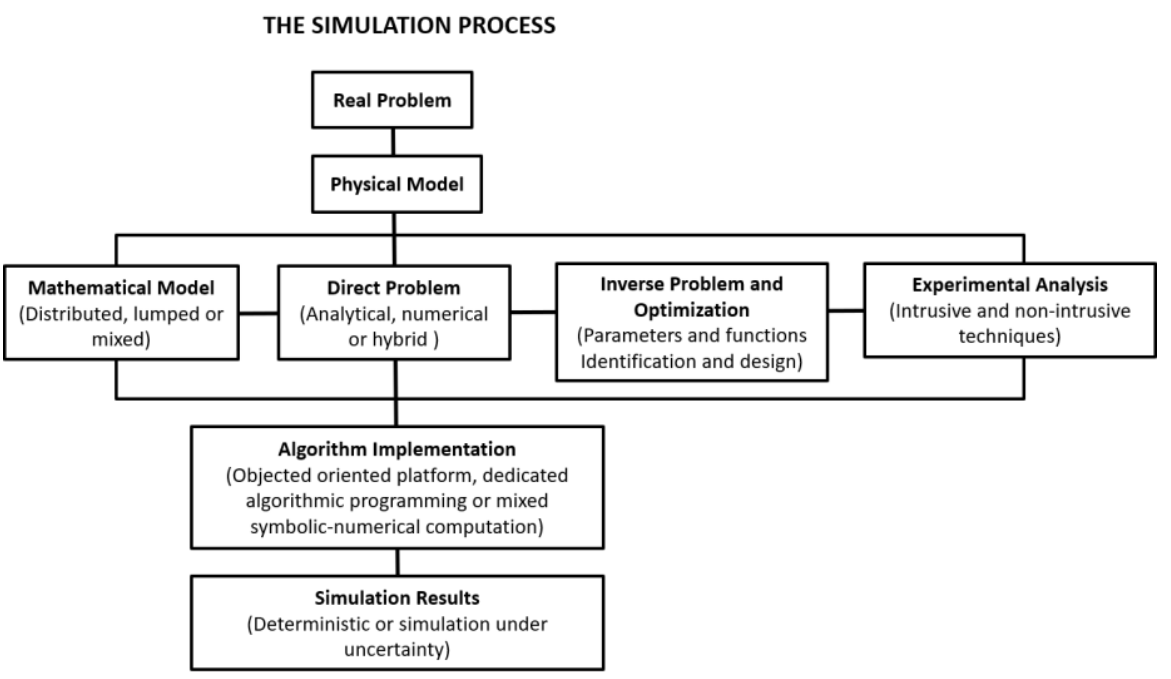

Fig. 1. Summary of the simulation process in engineering development.

Starting at the mathematical model stage, the hybrid approach is already handy in providing mixed lumped-differential formulations, specifically employed to solve diffusion and convection-diffusion problems. The idea behind the partial or total lumping of partial differential formulations lies in the elimination of space variables from the formulation through an averaging process, once the local information in that particular coordinate or coordinates is not essential to the problem solution. However, the process involves an approximation, whereby the local boundary information in the coordinate to be eliminated, which reappears within the partial differential equation during the averaging process, needs to be expressed analytically in terms of the averaged potential. The classical lumped system analysis assumes negligible gradients along that coordinate and equates the average and boundary potential values. This is in general not sufficiently accurate and is thus of limited use. Higher order approximations that provide a more informative relation between boundary and average potentials are thus quite useful in model reduction efforts. The Coupled Integral Equations Approach (CIEA) [14$20]$ is a systematic methodology for constructing improved lumped-differential formulations, based on approximating the integral expressions for the average potential and its associated flux through Hermite formulae for integration, which employ only the function and its spatial derivatives at the boundary points [21]. Then, more informative expressions relating average and boundary potentials are 
achieved, which are then incorporated in the averaged differential formulation. Accuracy is significantly improved over the classical lumping approach, while retaining the benefits such as the reductions in mathematical complexity and in simulation time/effort compared to the solution of the original fully differential problem formulation. Also of great relevance in the mathematical modeling stage is the reformulation of problems in heterogeneous media and/or complex geometrical arrangements as a single domain mathematical problem, which can be essential for a proper hybrid numerical-analytical treatment. The concept of a single domain formulation was formalized in the context of natural convection in porous media and conjugated heat transfer problems [22-25], and progressively employed to handle other classes of problems. The idea is simply that of rewriting a system of coupled equations for a composite/multi-component system, with various common interfaces, as a single region in which the functions associated with physical properties and sources are rewritten in the form of coefficients with space variability, accounting for the transitions through the different phases or components.

The direct problem solution stage in any simulation process is by far the most extensively studied in the literature. The advent of the electronic computer brought together a revolution on the numerical analysis and simulation tools for partial differential equations, through the best-known numerical techniques such as finite difference, finite element, and finite volume methods [26], which markedly changed the advanced engineering practice. Nevertheless, after decades of substantial scientific and technological progress on both software and hardware, such methodologies are still not universal or fully reliable, despite the considerable efforts of extension, generalization, and automatic error control. Particularly, from a computational cost perspective, they yet remain quite demanding and even prohibitive in computationally intensive tasks. As examples of situations when the direct problem solution needs to be obtained several thousands of times, one can recall the identification of parameters and functions in inverse problems analysis, the geometrical and parametric optimization of process performance, and the stochastic analysis of uncertainty propagation. While the classical analytical approaches for partial differential equations can be very restrictive in terms of classes of problems that can be dealt with, over the last three decades, hybrid numerical-analytical approaches have been developed in different physical contexts, at a much more modest pace in comparison to purely numerical methods, based on different analytical tools. The Generalized Integral Transform Technique (GITT), based on the classical integral transform method [27-29], has introduced a hybrid nature to this eigenfunction expansion approach [30-32], yielding errorcontrolled solutions to a large number of linear and nonlinear convection-diffusion problems, as reviewed in various sources [33-39]. The interest in analytical solution methodologies for PDE's might not be so evident to the less experienced researcher in the field, but it has been experiencing a noticeable revival, mainly for the following reasons:

1. Exact solutions: analytical methods lead to computationally costless solutions, with user prescribed accuracy, though such solutions might not be 
obtainable when the formulation complexity increases;

2. Trends and asymptotic behaviors: analytically obtained solutions offer minimal computational effort, besides yielding parametric analysis and limiting behavior identification even with very little computational work;

3. Reference benchmark results: independent analytical benchmark results are essential in verification of numerical methods and codes;

4. More straightforward and feasible: symbolic computation platforms have enabled a number of ideas on analytically-based methodologies that were either abandoned or never even considered;

5. Intensive computational tasks: the advantage of analytical solutions are even more evident in costly computations, such as parameters and functions inverse analysis identification, process optimization, and stochastic simulation for uncertainty propagation;

6. Bridge to build hybrid numerical-analytical approaches: they are instrumental in the search for higher precision and robustness in classes of problems that are not solvable through classical analytical methods.

The basic steps in the GITT approach can be summarized as follows [33-39]:

1. Select an analytical filtering solution: to improve convergence behavior of the eigenfunction expansions, if required. Filtering to achieve homogeneous boundary conditions and/or reduce the importance of equation source terms is always helpful. Filtering can also be applied recursively if necessary, or implicitly, such as in the case of a nonlinear filter.

2. Choose the associated eigenvalue problem: which should desirably incorporate characteristic linear behaviors of the original problem formulation represented by the coefficients of the differential operators. Both diffusive or convective eigenvalue problems may be adopted. More recently, nonlinear eigenvalue problems have also been employed with marked improvement on convergence.

3. Develop the integral transform pair: obtain the transform and inversion, that define the integral transform operation and explicitly recover the potential.

4. Solve the eigenvalue problem: either in analytical form and symbolic computation [40], or through the GITT approach itself, transforming the chosen differential eigenvalue problem into an algebraic one.

5. Integral transform the original PDE: obtain the transformed differential system, which shall be an ODE system for a total transformation, when all the independent variables are eliminated except one. It can result in an initial value problem, for an original parabolic or hyperbolic-type problem, or in a boundary value problem, for an original elliptic formulation. A partial transformation may also be applied, when two independent variables are kept in the transformed system, yielding a partial differential transformed system. The partial transformation option may also be adopted in solving a boundary value problem via a pseudo-transient formulation.

6. Compute transformed system coefficients: the coefficients are integrals, 
single or multiple, which involve the eigenfunctions. When analytical expressions are not obtainable through symbolic computation [40], there is a marked advantage in promoting semi-analytical integrations, when the oscillatory nature of the eigenfunctions is analytically handled while the nonoscillatory portion of the integrand is approximated by piecewise polynomial interpolation. Traditional numerical integration may also be employed and, again, the knowledge about the oscillatory behavior of the eigenfunctions can be advantageous in defining appropriate domain partitions to reduce computational costs in adaptive integration, especially for higher order eigenvalues, which represent the higher oscillation frequencies.

7. Solve transformed system: numerically (or analytically when applicable) solve the resulting coupled ODE or PDE system for the transformed potentials, which needs to be truncated to a sufficiently large order for numerical evaluation. Reliable automatic solvers are readily available for the numerical solution of stiff ODE systems, and even for one-dimensional systems of PDE's with adaptive remeshing, such as in the routine NDSolve of the Mathematica system [40];

8. Recall inversion formula: to analytically reconstruct the hybrid solution of the desired potential. At this point, the inversion formula can be employed for accuracy testing, once the intermediate tasks were accomplished under error control, and thus allowing for best selection of the transformed system truncation orders. Error estimates are then automatically provided at any desired position and time.

This hybrid approach has been extensively used as a benchmarking tool, aiding in the verification of more general and flexible numerical codes, but also as a production tool itself, as previously discussed, especially in highly intensive computational jobs and/or precision demanding applications. The various classes of problems so far treated by the GITT include linear and nonlinear diffusion, convection-diffusion, and reaction-convection-diffusion problems, moving boundary problems, eigenvalue problems of unknown analytical solution, irregular domains, heterogeneous media, boundary layer equations, and Navier-Stokes equations [30-39]. Fairly recently, an open source general purpose symbolicnumerical code was implemented, coined as the UNIT code (Unified Integral Transforms) [41-42] and implemented in the Mathematica platform [40]. It offers all of the basic analytical and numerical steps in the GITT implementation, allowing for a more straightforward utilization of this methodology.

The next stage in the simulation process involves using both the direct problem solution and the experimental data from the adjacent stage in Fig.1, to perform the inverse problem analysis, as required, for parameter and function identification or even state estimation [43-44]. However, this analysis may also be employed ahead of the experimentation, aimed at achieving an optimal design for the experimental procedure and setup [43]. In the identification of properties and source terms, the direct problem solution is employed first for the sensitivity analysis of the quantities to be estimated and to produce simulated data, so as to evaluate the inverse problem 
solution ahead of employing actual experimental data. Bayesian inference has been preferred for the inverse analysis in the present context [45-52], first of all due to its inherent robustness, but also due to advantages in being able to account for a priori information on the parameters. The drawback of this statistical approach, associated with the usually large number of evaluations of the direct problem required for an appropriate statistical inference through the Monte Carlo Markov Chain method, is alleviated by the use of the present hybrid solution pathways with reduced computational cost. Also, in two other aspects the hybrid direct problem solution is particularly beneficial to the cost reduction in the inverse problem analysis. Firstly, the experimental data acquired by the non-intrusive experimental techniques, to be discussed later, is often available in large quantities, in both space and time, resolving a certain region in the overall domain in great detail. Therefore, it is feasible and interesting to work within the integral transformed domain, by performing the same integral transformation process of the direct problem solution, onto the spatially distributed experimental data. Such is the case, for example, with temperature measurement fields obtained by infrared thermography (IRT) or laser induced fluorescence (LIF) methods, velocity and concentration distributions obtained with particle image velocimetry (PIV) and LIF methods. A marked data reduction effect then occurs, with an additional regularization effect on the inverse problem solution. Secondly, for a function estimation process, the unknown function can itself be expressed as an eigenfunction expansion, as a choice of parametrization, which permits obtaining analytical expressions for the transformed system coefficients, thus further reducing computational costs by avoiding numerical integration at the direct problem solution stage. Figure 2 summarizes the interconnections that characterize the hybrid approach advocated here, involving the four stages of mathematical model, direct problem, inverse problem, and experiments.

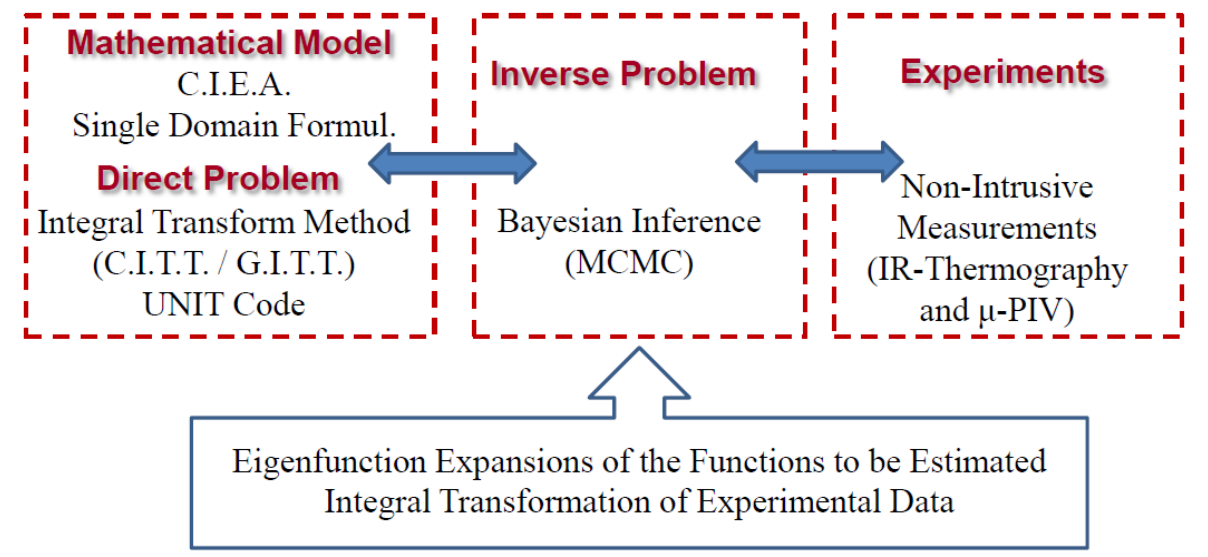

Fig. 2. The hybrid approach in mathematical modeling and direct-inverse problem analysis. 
In parallel to the advancement of inverse problem analysis tools, experimental techniques have experienced significant progresses along recent years, such as in the case of infrared thermography for non-intrusive temperature measurements [5359]. Microscopic lenses can also be employed to refine further the spatial information, and pixels of the order of 20 microns are fairly common in the present commercial IRT systems. However, these temperature measurements are in general restricted to external surfaces of the micro-systems, unless IR transparent windows are employed to capture the internal fluid interface temperatures. Nevertheless, the inverse problem analysis may provide estimates of internal temperatures, working as a virtual sensor. Other non-intrusive experimental techniques have been employed in the context of this joint research effort, such as Micro particle image velocimetry $(\mu \mathrm{PIV})$ for velocity measurements and Micro laser induced fluorescence $(\mu \mathrm{LIF})$ for temperature and concentration measurements [59-65]. $\mu \mathrm{PIV}$ is a non-intrusive quantitative imaging technique that uses statistical crosscorrelation algorithms to detect the particle motion of tracer particles seeded in the microscale flows. By determining the particle displacement in the flow, $\mu$ PIV enables measurements of the actual velocity field of the imaged microfluidic systems. Fluid temperature measurements in microscale devices can be obtained using a $\mu$ LIF system by means of a suitably selected fluorescent dye injected in the flow to sense local temperature.

The stage of algorithm implementation follows the simulation process shown schematically in Fig. 1. At least three possibilities are shown in the graph; namely the employment of an object-oriented platform, such as the well-known commercial multiphysics simulations software systems, the preparation of dedicated algorithmic codes through one of the well-established high level programming languages, and the use of modern symbolic computation environments. All of these tools add up to a successful engineering development effort. In the present research, particular emphasis is given on the mixed symbolic-numerical Mathematica platform [40].

\section{APPLICATION TO MICROSCALE TRANSPORT PHENOMENA}

Microsystems based on flowing fluids have been extensively developed along the last few decades, including among others, micro-mixers, micro-pumps, micro-reactors, and micro-heat exchangers, benefiting from the larger surface area/volume ration aimed at enhancing and accelerating transport phenomena. Such technological development efforts have required advancing not only experimental techniques at the micro-scale, but also formulation and methodological extensions for the simulation of these micro-devices. While the continuum mechanics hypotheses are usually valid within the range of dimensions of such systems, there are sensible differences in the relative importance of the various possible effects in comparison to the more usual macro-systems. For instance, conjugated heat transfer with the device substrates, axial diffusion along the fluid currents, viscous dissipation effects, fluid 
slip in liquid flows over superhydrophobic surfaces, and flow rarefaction at the channel walls for gaseous flows, are examples of phenomena that may play a major role when modelling fluid flow and heat and mass transfer at the micro-scale. Since the computational analysis of transport phenomena in microsystems usually involves multiple scales in geometry and physical parameters, the costs associated with traditional numerical methods tend to markedly increase, thus making the present hybrid method more attractive in such classes of problems, as illustrated in [10].

In this chapter, the hybrid methodology will be illustrated by considering a microreactor analysis for continuous synthesis of biodiesel. The findings presented are part of an ongoing collaborative research aiming to improve the design of the microreactors recently demonstrated for biodiesel synthesis [66], targeting residence times of around one minute or less. For this purpose, the hybrid approach has been employed throughout the development and design of this microfluidic system [67-71], then validated through experimental tests employing high speed and thermal imaging infrared cameras [72]. Advanced additive micro-manufacturing platforms were also used to propose a more economical fabrication of multiple microreactors to assemble a modular prototype [72]. There are key process parameters, such as oil and alcohol flow rates, geometry, concentration and type of catalyst, and substrate temperature, that can be optimized to reach increased efficiency of the basic unit within the prototype, which will then lead to the parallel arrangement of a sufficiently large number of microreactors for demonstration. It has also been employed to develop an innovative device for recovery of rejected heat from other processes, such as combustion engines or high concentration photovoltaic panels [73], through an integrated micro-heat exchanger mounted on the same microreactor substrate. For the sake of brevity, these aspects are not fully addressed here.

\subsection{Problem Formulation}

Biodiesel is a biodegradable product that can be used as an alternative fuel since its physico-chemical properties are very similar to those of conventional diesel, but with the advantages of being a clean and renewable source of energy and contributing to the reduction of pollution and greenhouse effect. The most common biodiesel production route is via the transesterification reaction that occurs in three successive steps [74]. Fatty acid esters (biodiesel) are obtained in each step of the reaction, ethyl ester in case ethanol is used as the alcohol, and methyl ester if methanol is used [74]. The three steps of the transesterification reaction are then written as:

$$
\begin{aligned}
& \text { Triglyceride }(T G)+\operatorname{Alcohol}(A) \underset{\mathrm{k}_{2}}{\stackrel{\mathrm{k}_{1}}{\rightleftarrows}} \operatorname{Diglyceride}(\mathrm{DG})+\operatorname{Ester}(\mathrm{B}) \\
& \operatorname{Diglyceride}(\mathrm{DG})+\operatorname{Alcohol}(\mathrm{A}) \underset{\mathrm{k}_{4}}{\stackrel{\mathrm{k}_{3}}{\rightleftarrows}} \operatorname{Monoglyceride}(\mathrm{G})+\operatorname{Ester}(\mathrm{B}) \\
& \operatorname{Monoglyceride}(\mathrm{MG})+\operatorname{Alcohol}(\mathrm{A}) \underset{\mathrm{k}_{6}}{\stackrel{\mathrm{k}_{5}}{\rightleftarrows}} \operatorname{Glycerol}(\mathrm{GL})+\operatorname{Ester}(\mathrm{B})
\end{aligned}
$$


where $\mathrm{k}_{1}, \mathrm{k}_{2}, \mathrm{k}_{3}, \mathrm{k}_{4}, \mathrm{k}_{5}$ and $\mathrm{k}_{6}$ are the kinetics coefficients that need to be determined for the proper simulation of the biodiesel synthesis process.

The design of microdevices for biodiesel production via transesterification reaction is a complicated task due to the complex liquid-liquid reactant flow that takes place within the microchannels [75-78]. Thus, the computational simulation is an important tool that helps to understand the physical-chemical phenomenon and, consequently, to determine the suitable conditions that maximize the conversion of triglycerides during the biodiesel synthesis. The mathematical model is obtained through a steady-state mass balance that considers diffusive-convectivereactive effects to describe the transesterification route for biodiesel synthesis in a microreactor of rectangular section [67-72], as represented in Figure 3.

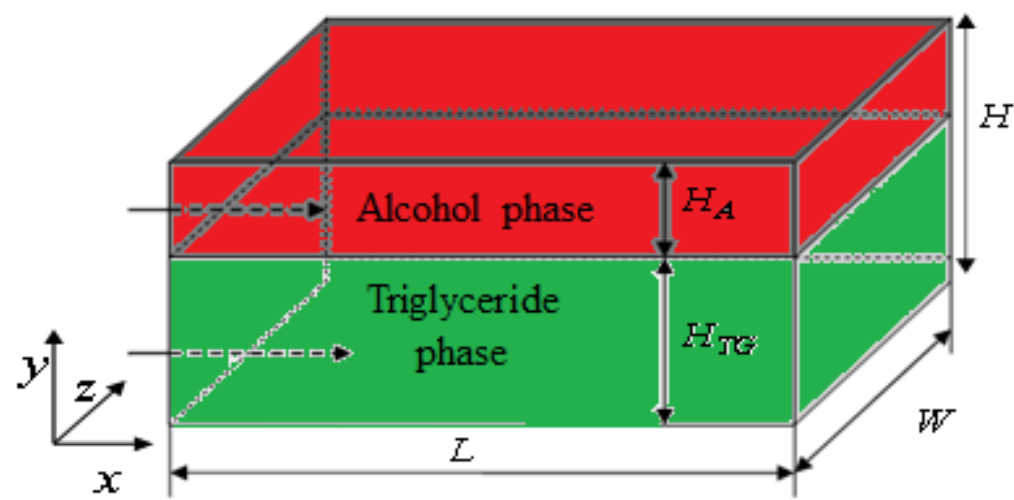

Fig. 3. Geometry and coordinates system of micro-reactor for biodiesel synthesis (triglyceride - green region, alcohol+catalyst - red region).

The dimensionless mass transfer equations for the species concentrations, considering second order consecutive elementary and reversible reactions preferably in the triglyceride phase, under isothermal conditions and with constant physical properties, can be written as:

$$
\begin{gathered}
U_{T G}(Y, Z) \frac{\partial F_{s}(X, Y, Z)}{\partial X}=\frac{1}{P e_{s}} \frac{\partial^{2} F_{s}(X, Y, Z)}{\partial X^{2}}+\xi_{s}\left(\frac{\partial^{2} F_{s}(X, Y, Z)}{\partial Y^{2}}+\delta \frac{\partial^{2} F_{s}(X, Z)}{\partial Z^{2}}\right)+\varsigma G_{s} \\
F_{T G}(0, Y, Z)=1 ; \quad F_{s}(0, Y, Z)=0, \quad s=\mathrm{A}, \mathrm{DG}, \mathrm{MG}, \mathrm{GL}, \mathrm{B} \\
\left.\frac{\partial F_{s}}{\partial X}\right|_{X=1}=\left.\frac{\partial F_{s}}{\partial Y}\right|_{Y=0}=\left.\frac{\partial F_{s}}{\partial Z}\right|_{Z=0}=\left.\frac{\partial F_{s}}{\partial Z}\right|_{Z=1}=0, \text { for all species } s \\
F_{A}(X, 1, Z)=F_{A_{0}} ;\left.\quad \frac{\partial F_{s}}{\partial Y}\right|_{Y=1}=0, \quad s=\mathrm{TG}, \mathrm{DG}, \mathrm{MG}, \mathrm{GL}, \mathrm{B}
\end{gathered}
$$

where $F_{s}$ are the dimensionless concentrations and the kinetic expressions for each species, $G_{s}$, are given by [69]: 


$$
\begin{gathered}
G_{T G}=-k_{1} F_{T G} F_{A}+k_{2} F_{D G} F_{B} \\
G_{A}=\left(-k_{1} F_{T G}-k_{3} F_{D G}-k_{5} F_{M G}\right) F_{A}+\left(k_{2} F_{D G}+k_{4} F_{M G}+k_{6} F_{G L}\right) F_{B} \\
G_{D G}=\left(k_{1} F_{T G}-k_{3} F_{D G}\right) F_{A}+\left(-k_{2} F_{D G}+k_{4} F_{M G}\right) F_{B} \\
G_{M G}=\left(k_{3} F_{D G}-k_{5} F_{M G}\right) F_{A}+\left(-k_{4} F_{M G}+k_{6} F_{G L}\right) F_{B} \\
G_{G L}=k_{5} F_{M G} F_{A}-k_{6} F_{G L} F_{B} \\
G_{B}=\left(k_{1} F_{T G}+k_{3} F_{D G}+k_{5} F_{M G}\right) F_{A}+\left(-k_{2} F_{D G}-k_{4} F_{M G}-k_{6} F_{G L}\right) F_{B}
\end{gathered}
$$

while the velocity profiles for the vegetable oil and the alcohol, assumed fully developed and in laminar stratified regime, are given by [69]:

$$
\begin{aligned}
& u_{T G}(y, z)=\sum_{i=1}^{\infty}\left(\frac{2}{w}\right)^{1 / 2} \sin \left(\frac{i \pi}{w} z\right) S_{i}\left\{\left(1+\frac{\mu_{A}}{\mu_{T G}}\right)\left[-\sinh \left(\frac{H i \pi}{w}\right)+\sinh \left(\frac{i \pi(H-y)}{w}\right)\right]+\right. \\
& +\left(\frac{\mu_{A}}{\mu_{T G}}-1\right)\left[\sinh \left(\frac{\left(H-2 H_{T G}\right) i \pi}{w}\right)+\sinh \left(\frac{i \pi\left(H-H_{T G}+y\right)}{w}\right)+\right. \\
& \left.\left.-\sinh \left(\frac{i \pi\left(H-H_{T G}-y\right)}{w}\right)-\sinh \left(\frac{i \pi\left(H-2 H_{T G}+y\right)}{w}\right)\right]+2 \sinh \left(\frac{i \pi y}{w}\right)\right\} \\
& \quad u_{A}(y, z)=\sum_{i=1}^{\infty}\left(\frac{2}{w}\right)^{1 / 2} \sin \left(\frac{i \pi}{w} z\right) S_{i}\left\{\left(1+\frac{\mu_{T G}}{\mu_{A}}\right)\left[-\sinh \left(\frac{H i \pi}{w}\right)+\sinh \left(\frac{i \pi y}{w}\right)\right]+\right. \\
& \quad+\left(1-\frac{\mu_{T G}}{\mu_{A}}\right)\left[\sinh \left(\frac{\left(H-2 H_{T G}\right) i \pi}{w}\right)+\sinh \left(\frac{i \pi\left(2 H_{T G}-y\right)}{w}\right)+\right. \\
& \left.\left.\quad-\sinh \left(\frac{i \pi\left(H-H_{T G}-y\right)}{w}\right)-\sinh \left(\frac{i \pi\left(H+H_{T G}-y\right)}{w}\right)\right]+2 \sinh \left(\frac{i \pi(H-y)}{w}\right)\right\}
\end{aligned}
$$

with $i=1,3,5, \ldots$ and

$$
S_{i}=\frac{-2 \sqrt{2} w^{5 / 2} \Delta P}{i^{3} L \pi^{3}\left[\left(\mu_{T G}-\mu_{A}\right) \sinh \left(\frac{\left(H-2 H_{T G}\right) i \pi}{w}\right)+\left(\mu_{T G}+\mu_{A}\right) \sinh \left(\frac{H i \pi}{w}\right)\right]}
$$

The associated dimensionless groups are given by:

$$
\begin{aligned}
& F_{T G}=\frac{C_{T G}}{C_{T G o}} ; F_{A}=\frac{C_{A}}{C_{T G o}} ; F_{D G}=\frac{C_{D G}}{C_{T G o}} ; F_{M G}=\frac{C_{M G}}{C_{T G o}} ; F_{B}=\frac{C_{B}}{C_{T G o}} ; F_{G L}=\frac{C_{G L}}{C_{T G o}} ; \\
& F_{A o}=\frac{C_{A}^{*}}{C_{T G o}} ; Y=\frac{y}{H_{T G}} ; X=\frac{x}{L} ; Z=\frac{z}{w} ; U_{T G}(Y, Z)=\frac{u_{T G}(Y, Z)}{u_{T G, A V}} ; \varsigma=\frac{L C_{T G o}}{u_{T G, A V}} ;
\end{aligned}
$$




$$
\xi_{s}=\frac{L D_{s}}{u_{T G, A V} H_{T G}^{2}} ; P e_{s}=\frac{u_{T G, A V} L}{D_{s}} ; \delta=\frac{H_{T G}^{2}}{w^{2}}
$$

where $C$ 's and $D$ 's are the dimensional concentrations and the mass diffusion coefficients of each species, $u_{T G, A V}$ is the average flow velocity for the layer containing the triglyceride species, $\mu$ represents the dynamic viscosities of the fluids, $H$ is height of the microchannel, $H_{T G}$ is the height of the fluids interface, $L$ represents the length and $\mathrm{W}$ the width of the microreactor, and $\Delta P$ is the total pressure drop along the microreactor.

$H_{T G}$, the height of triglyceride layer, can be numerically computed for specific experimental values from the ratio between the volumetric flow rates of the two phases present in the microsystem (triglyceride and alcohol), using the following relation:

$$
\frac{Q_{T G}}{Q_{A}}=\frac{\int_{0}^{w} \int_{0}^{H_{T G}} u_{T G}(y, z) d y d z}{\int_{0}^{w} \int_{H_{T G}}^{H} u_{A}(y, z) d y d z}
$$

where $Q_{T G}$ and $Q_{A}$ are the triglyceride and the alcohol volumetric flow rates, respectively, while the pressure drop $\Delta P$ is obtained from the equation for the volumetric flow rate of the triglyceride phase.

In order to evaluate the influence of temperature on the reaction conversion rate under isothermal conditions, the Arrhenius equation can be used to estimate the kinetic constants at different temperature levels, as follows [70]:

$$
k_{i, 1}=\alpha_{i} \operatorname{Exp}\left[-\frac{E_{i}}{R T_{1}}\right], \quad i=1,2, \ldots, 6
$$

where $k_{i, 1}$ is the kinetic constant at the temperature $T_{1}$. Manipulating eq. (5a), the ratio of the kinetic constants at different temperature levels can be readily obtained from:

$$
\ln \left(\frac{k_{i, 2}}{k_{i, 1}}\right)=\frac{E_{i}}{R}\left[\frac{1}{T_{1}}-\frac{1}{T_{2}}\right], \quad i=1,2, \ldots, 6
$$

where $k_{i, 2}$ is the kinetic constant at the temperature $T_{2}$.

The consumption of triglyceride along the biodiesel synthesis is influenced by the time the species remain inside the micro-channel, also called the residence time. Since it is assumed that the reaction occurs only in the triglyceride phase, then the residence 
time may be determined from the ratio of the triglyceride layer volume and its volumetric flow rate, as:

$$
\tau=\frac{L W H_{T G}}{Q_{T G}}
$$

The triglyceride consumption is evaluated in terms of the fractional conversion, according to:

$$
\text { ConversionTG(\%)=( } \left.\frac{F_{T G, A V}(0)-F_{T G, A V}(1)}{F_{T G, A V}(0)}\right) \times 100
$$

\subsection{GITT Solution of the 3D Microreactor Problem}

Equations (1) constitute a nonlinear system of three-dimensional partial differential equations, coupled through the kinetic expressions for each species, which has been solved by the Generalized Integral Transform Technique (GITT) [69-70]. First of all, in order to homogenize the boundary condition of the alcohol species in the $Y$ direction and ensure improved convergence behavior, a simple filter is proposed as:

$$
F_{A}(X, Y, Z)=F_{A h}(X, Y, Z)+F_{A o}
$$

To satisfy the boundary conditions and establish the basis to represent the concentrations as eigenfunction expansions, two different eigenvalue problems were adopted, one for the alcohol species and the other for the remaining species. In [70], two different possibilities were investigated, either accounting for the velocity profile in the eigenvalue problem or just considering the simplest constant coefficients formulation. Here, only the second alternative is detailed to illustrate the methodology, as in [69], but results are compared later on for both solution paths. Thus, the eigenvalue problem for the alcohol concentration equation can be defined as:

$$
\begin{gathered}
\frac{\partial^{2} \Gamma_{i}}{\partial Y^{2}}+\delta \frac{\partial^{2} \Gamma_{i}}{\partial Z^{2}}+\beta_{i}^{2} \Gamma_{i}(Y, Z)=0 \\
\left.\frac{\partial \Gamma_{i}}{\partial Y}\right|_{Y=0}=0, \Gamma_{i}(1, Z)=0,\left.\frac{\partial \Gamma_{i}}{\partial Z}\right|_{Z=0}=0,\left.\frac{\partial \Gamma_{i}}{\partial Z}\right|_{Z=1}=0
\end{gathered}
$$

Problem (8) is then solved by Separation of Variables, leading to a couple of onedimensional problems to represent the original eigenfunction $\Gamma(Y, Z)$ in accordance with Table 1 below. One can then express the normalized eigenfunction $\tilde{\Gamma}(Y, Z)$ and the corresponding eigenvalues as: 


$$
\begin{gathered}
\tilde{\Gamma}_{m, n}(Y, Z)=\tilde{\phi}_{1, m}(Y) \tilde{\phi}_{2, n}(Z) \\
\beta_{m, n}^{2}=\beta_{1, m}^{2}+\beta_{2, n}^{2}
\end{gathered}
$$

Table 1. Auxiliary eigenvalue problems for eigenfunction $\Gamma(Y, Z)$ :

a) Eigenvalue problems; b) Normalized eigenfunction; c) Norm; d) Eigenvalues

\begin{tabular}{|l|c|c|}
\hline & Coordinate $Y$ & Coordinate $Z$ \\
\hline a) & $\frac{d^{2} \phi_{1, m}}{d Y^{2}}+\beta_{1, m}^{2} \phi_{1, m}(Y)=0$ & $\delta \frac{d^{2} \phi_{2, n}}{d Z^{2}}+\beta_{2, n}^{2} \phi_{2, n}(Z)=0$ \\
& $\left.\frac{d \phi_{1, m}}{d Y}\right|_{Y=0}=0, \phi_{1, m}(1)=0$ & $\left.\frac{d \phi_{2, n}}{d Z}\right|_{Z=0}=0,\left.\frac{d \phi_{2, n}}{d Z}\right|_{Z=1}=0$ \\
\hline b) & $\tilde{\phi}_{1, m}(Y)=\frac{\cos \left(\beta_{1, \mathrm{~m}} Y\right)}{\sqrt{N_{\phi 1}}}$ & $\tilde{\phi}_{2, n}(Z)=\frac{1}{\sqrt{N_{\phi 2}}}\left\{\begin{array}{c}1 \\
\cos \left(\frac{\beta_{2, n}}{\sqrt{\delta}} Z\right) \text { if } \beta_{2, n}=0\end{array} \beta_{2, n} \neq 0\right.$ \\
\hline c) & $N_{\phi 1}=\int_{0}^{1} \phi_{1, m}^{2}(Y) d Y=\frac{1}{2}$ & $N_{\phi 2}=\int_{0}^{1} \phi_{2, m}^{2}(Y) d Z= \begin{cases}1 & \text { if } \beta_{2, n}=0 \\
1 / 2 & \text { if } \beta_{2, n} \neq 0\end{cases}$ \\
\hline d) & $\beta_{1, m}=\frac{(2 m-1) \pi}{2}, m=1,2, \ldots$ & $\beta_{2, \mathrm{n}}=\sqrt{\delta} n \pi, n=0,1,2, \ldots$ \\
\hline
\end{tabular}

The eigenvalue problem for the other remaining species can be defined as:

$$
\begin{gathered}
\frac{\partial^{2} \Psi_{i}}{\partial Y^{2}}+\delta \frac{\partial^{2} \Psi_{i}}{\partial Z^{2}}+\mu_{i}^{2} \Psi_{i}(Y, Z)=0 \\
\left.\frac{\partial \Psi_{i}}{\partial Y}\right|_{Y=0}=0,\left.\frac{\partial \Psi_{i}}{\partial Y}\right|_{Y=1}=0,\left.\frac{\partial \Psi_{i}}{\partial Z}\right|_{Z=0}=0,\left.\frac{\partial \Psi_{i}}{\partial Z}\right|_{Z=1}=0
\end{gathered}
$$

Again, problem (10) is readily solved analytically and the solution is expressed through the one-dimensional eigenvalue problems as presented in Table 2. Then, the normalized eigenfunction $\tilde{\Psi}$ and its eigenvalues can be written as:

$$
\begin{gathered}
\tilde{\Psi}_{m, n}(Y, Z)=\tilde{\varphi}_{1, m}(Y) \tilde{\varphi}_{2, n}(Z) \\
\mu_{m, n}^{2}=\mu_{1, m}^{2}+\mu_{2, n}^{2}
\end{gathered}
$$


Table 2. Auxiliary eigenvalue problems for eigenfunction $\Psi(Y, Z)$ :

a) Eigenvalue problems; b) Normalized eigenfunction; c) Norm; d) Eigenvalues

\begin{tabular}{|c|c|c|}
\hline & Coordinate $Y$ & Coordinate $Z$ \\
\hline a) & $\begin{array}{c}\frac{d^{2} \varphi_{1, m}}{d Y^{2}}+\mu_{1, m}^{2} \varphi_{1, m}(Y)=0 \\
\left.\frac{d \varphi_{1, m}}{d Y}\right|_{Y=0}=0,\left.\frac{d \varphi_{1, m}}{d Y}\right|_{Y=1}=0\end{array}$ & $\begin{array}{l}\delta \frac{d^{2} \varphi_{2, n}}{d Y^{2}}+\mu_{2, n}^{2} \varphi_{2, n}(Y)=0 \\
\left.\frac{d \varphi_{2, n}}{d Y}\right|_{Y=0}=0,\left.\frac{d \varphi_{2, n}}{d Y}\right|_{Y=1}=0\end{array}$ \\
\hline b) & $\tilde{\varphi}_{1, m}(Y)=\frac{1}{\sqrt{N_{\varphi 1}}}\left\{\begin{array}{c}1 \quad \text { if } \mu_{1, m}=0 \\
\cos \left(\mu_{1, m} Y\right) \text { if } \mu_{1, m} \neq 0\end{array}\right.$ & $\tilde{\varphi}_{2, n}(Z)=\frac{1}{\sqrt{N_{\varphi 2}}}\left\{\begin{array}{cc}1 & \text { if } \mu_{2, n}=0 \\
\cos \left(\frac{\mu_{2, n}}{\sqrt{\delta}} Z\right) & \text { if } \mu_{2, n} \neq 0\end{array}\right.$ \\
\hline c) & $N_{\varphi 1}=\int_{0}^{1} \varphi_{1, m}^{2}(Y) d Y= \begin{cases}1 & \text { if } \mu_{1, \mathrm{~m}}=0 \\
1 / 2 & \text { if } \mu_{1, \mathrm{~m}} \neq 0\end{cases}$ & $N_{\varphi 2}=\int_{0}^{1} \varphi_{2, m}^{2}(Z) d Z= \begin{cases}1 & \text { if } \mu_{2, n}=0 \\
1 / 2 & \text { if } \mu_{2, n} \neq 0\end{cases}$ \\
\hline d) & $\mu_{1, m}=m \pi, m=0,1,2, \ldots$ & $\mu_{2, n}=\sqrt{\delta} n \pi, n=0,1,2, \ldots$ \\
\hline
\end{tabular}

After solving the related eigenvalue problems, the following integral transform pairs for each of the species involved in the transesterification reaction can be defined:

- Transforms

$$
\begin{aligned}
& \bar{F}_{T G_{i}}(X)=\int_{0}^{1} \int_{0}^{1} \tilde{\Psi}_{i}(Y, Z) F_{T G}(X, Y, Z) d Y d Z \\
& \bar{F}_{A h_{i}}(X)=\int_{0}^{1} \int_{0}^{1} \tilde{\Gamma}_{i}(Y, Z) F_{A h}(X, Y, Z) d Y d Z \\
& \bar{F}_{D G_{i}}(X)=\int_{0}^{1} \int_{0}^{1} \tilde{\Psi}_{i}(Y, Z) F_{D G}(X, Y, Z) d Y d Z \\
& \bar{F}_{M G_{i}}(X)=\int_{0}^{1} \int_{0}^{1} \tilde{\Psi}_{i}(Y, Z) F_{M G}(X, Y, Z) d Y d Z \\
& \bar{F}_{B_{i}}(X)=\int_{0}^{1} \int_{0}^{1} \tilde{\Psi}_{i}(Y, Z) F_{B}(X, Y, Z) d Y d Z \\
& \bar{F}_{G L_{i}}(X)=\int_{0}^{1} \int_{0}^{1} \tilde{\Psi}_{i}(Y, Z) F_{G L}(X, Y, Z) d Y d Z
\end{aligned}
$$


- Inverses

$$
\begin{aligned}
& F_{T G}(X, Y, Z)=\sum_{i=1}^{\infty} \tilde{\Psi}_{i}(Y, Z) \bar{F}_{T G_{i}}(X), F_{A h}(X, Y, Z)=\sum_{i=1}^{\infty} \tilde{\Gamma}_{i}(Y, Z) \bar{F}_{A h_{i}}(X) \\
& F_{D G}(X, Y, Z)=\sum_{i=1}^{\infty} \tilde{\Psi}_{i}(Y, Z) \bar{F}_{D G_{i}}(X), F_{M G}(X, Y, Z)=\sum_{i=1}^{\infty} \tilde{\Psi}_{i}(Y, Z) \bar{F}_{M G_{i}}(X)(13 \mathrm{a}-\mathrm{f}) \\
& F_{B}(X, Y, Z)=\sum_{i=1}^{\infty} \tilde{\Psi}_{i}(Y, Z) \bar{F}_{B_{i}}(X), F_{G L}(X, Y, Z)=\sum_{i=1}^{\infty} \tilde{\Psi}_{i}(Y, Z) \bar{F}_{G L_{i}}(X)
\end{aligned}
$$

It can be noticed that the eigenfunction expansions in eqs. (13) have been represented with a single index $i$, thus combining the eigenvalues and eigenfunctions of the one-dimensional auxiliary problems by reordering the summation terms in decreasing order of importance, adopting the sum of the squared eigenvalues as the sorting criterium [16,69]. Finally, the integral transformation of eqs. (1a-o), after filtering with eq.(7), is accomplished employing the integral transform operator with the respective eigenfunction for each species along both the $Y$ and $Z$ domains. The transformed ODE system then becomes:

$$
\begin{gathered}
\sum_{j=1}^{\infty} O_{i j}^{1} \frac{d \bar{F}_{s_{j}}}{d X}=\frac{1}{P e_{s}} \frac{d^{2} \bar{F}_{s_{j}}}{d X^{2}}-\xi_{s} \mu_{i}^{2} \bar{F}_{s_{i}}+\varsigma \bar{G}_{s, i j k}, \text { where } s=T G, D G, M G, B, G L \\
\sum_{j=1}^{\infty} O_{i j}^{2} \frac{d \bar{F}_{A h_{j}}}{d X}=\frac{1}{P e_{A}} \frac{d^{2} \bar{F}_{A h_{j}}}{d X^{2}}-\xi_{A} \beta_{i}^{2} \bar{F}_{A h_{i}}+\varsigma \bar{G}_{A h, i j k} \\
\bar{F}_{T G_{i}}(0)=\bar{f}_{1, i}, \bar{F}_{A h_{i}}(0)=-F_{A o} \bar{f}_{2, i}, \bar{F}_{s_{i}}(0)=0, \text { where } s=D G, M G, B, G L \\
\left.\frac{d \bar{F}_{s_{i}}}{d X}\right|_{X=1}=0, \text { for all species } s
\end{gathered}
$$

The term $\bar{G}_{s, i j k}$ represents the transformed reaction kinetic terms, according to:

$$
\begin{gathered}
\bar{G}_{T G, i j k}=\sum_{k=1}^{\infty} \sum_{j=1}^{\infty}\left(-k_{1} O_{i j k}^{3} \bar{F}_{T G_{j}} \bar{F}_{A h_{k}}+k_{2} O_{i j k}^{4} \bar{F}_{D G_{j}} \bar{F}_{B_{k}}\right)-k_{1} F_{A o} \bar{F}_{T G_{i}} \\
\bar{G}_{A, i j k}=\sum_{k=1}^{\infty} \sum_{j=1}^{\infty}\left[O_{i j k}^{5}\left(-k_{1} \bar{F}_{T G_{j}}-k_{3} \bar{F}_{D G_{j}}-k_{5} \bar{F}_{M G_{j}}\right) \bar{F}_{A h_{k}}+\right. \\
\left.O_{i j k}^{6}\left(k_{2} \bar{F}_{D G_{j}}+k_{4} \bar{F}_{M G_{j}}+k_{6} \bar{F}_{G L_{j}}\right) \bar{F}_{B_{k}}\right]+F_{A o} \sum_{j=1}^{\infty} O_{i j}^{7}\left(-k_{1} \bar{F}_{T G_{j}}-k_{3} \bar{F}_{D G_{j}}-k_{5} \bar{F}_{M G_{j}}\right) \\
\bar{G}_{D G, i j k}=\sum_{k=1}^{\infty} \sum_{j=1}^{\infty}\left[O_{i j k}^{3}\left(k_{1} \bar{F}_{T G_{j}}-k_{3} \bar{F}_{D G_{j}}\right) \bar{F}_{A h_{k}}+O_{i j k}^{4}\left(-k_{2} \bar{F}_{D G_{j}}+k_{4} \bar{F}_{M G_{j}}\right) \bar{F}_{B_{k}}\right] \\
+F_{A o}\left(k_{1} \bar{F}_{T G_{i}}-k_{3} \bar{F}_{D G_{i}}\right)
\end{gathered}
$$




$$
\begin{gathered}
\bar{G}_{M G, i j k}=\sum_{k=1}^{\infty} \sum_{j=1}^{\infty}\left[O_{i j k}^{3}\left(k_{3} \bar{F}_{D G_{j}}-k_{5} \bar{F}_{M G_{j}}\right) \bar{F}_{A h_{k}}+O_{i j k}^{4}\left(-k_{4} \bar{F}_{M G_{j}}+k_{6} \bar{F}_{G L_{j}}\right) \bar{F}_{B_{k}}\right] \\
+F_{A o}\left(k_{3} \bar{F}_{D G_{i}}-k_{5} \bar{F}_{M G_{i}}\right) \\
\bar{G}_{G L, i j k}=\sum_{k=1}^{\infty} \sum_{j=1}^{\infty}\left(k_{5} O_{i j k}^{3} \bar{F}_{M G_{j}} \bar{F}_{A h_{k}}-k_{6} O_{i j k}^{4} \bar{F}_{G L_{j}} \bar{F}_{B_{k}}\right)+k_{5} F_{A o} \bar{F}_{M G_{i}} \\
\bar{G}_{B, i j k}=\sum_{k=1}^{\infty} \sum_{j=1}^{\infty}\left[O_{i j k}^{3}\left(k_{1} \bar{F}_{T G_{j}}+k_{3} \bar{F}_{D G_{j}}+k_{5} \bar{F}_{M G_{j}}\right) \bar{F}_{A h_{k}}+\right. \\
\left.+O_{i j k}^{4}\left(-k_{2} \bar{F}_{D G_{j}}-k_{4} \bar{F}_{M G_{j}}-k_{6} \bar{F}_{G L_{j}}\right) \bar{F}_{B_{k}}\right]+F_{A o}\left(k_{1} \bar{F}_{T G_{i}}+k_{3} \bar{F}_{D G_{i}}+k_{5} \bar{F}_{M G_{i}}\right)
\end{gathered}
$$

where the coefficients produced by the integral transformation procedure are given by:

$$
\begin{gathered}
O_{i j}^{1}=\int_{0}^{1} \int_{0}^{1} U_{T G} \tilde{\Psi}_{i} \tilde{\Psi}_{j} d Y d Z, O_{i j}^{2}=\int_{0}^{1} \int_{0}^{1} U_{T G} \tilde{\Gamma}_{i} \tilde{\Gamma}_{j} d Y d Z, O_{i j k}^{3}=\int_{0}^{1} \int_{0}^{1} \tilde{\Psi}_{i} \tilde{\Psi}_{j} \tilde{\Gamma}_{k} d Y d Z, \\
O_{i j k}^{4}=\int_{0}^{1} \int_{0}^{1} \tilde{\Psi}_{i} \tilde{\Psi}_{j} \tilde{\Psi}_{k} d Y d Z ; O_{i j k}^{5}=\int_{0}^{1} \int_{0}^{1} \tilde{\Gamma}_{i} \tilde{\Psi}_{j} \tilde{\Gamma}_{k} d Y d Z ; O_{i j k}^{6}=\int_{0}^{1} \int_{0}^{1} \tilde{\Gamma}_{i} \tilde{\Psi}_{j} \tilde{\Psi}_{k} d Y d Z \\
O_{i j}^{7}=\int_{0}^{1} \int_{0}^{1} \tilde{\Gamma}_{i} \tilde{\Psi}_{j} d Y d Z ; \bar{f}_{1, i}=\int_{0}^{1} \int_{0}^{1} \tilde{\Psi}_{i} d Y d Z ; \bar{f}_{2, i}=\int_{0}^{1} \int_{0}^{1} \tilde{\Gamma}_{i} d Y d Z
\end{gathered}
$$

Once the transformed concentrations have been numerically determined from solving the ODE system (14), the original concentrations can be recovered through the inverse formulas defined in eqs. (13a-f) and in the filter, eq. (7). The concentrations can also be presented in terms of flow averaged potentials, defined as follows:

$$
F_{\mathrm{s}, A V}(X)=\frac{\int_{0}^{1} \int_{0}^{1} U_{T G}(Y, Z) F_{s}(X, Y, Z) d Y d Z}{\int_{0}^{1} \int_{0}^{1} U_{T G}(Y, Z) d Y d Z}, s=T G, A, D G, M G, G L, B
$$

By introducing the eigenfunction expansions for the dimensionless concentrations of the species, the following average concentrations can be obtained:

$$
F_{s, A V}(X)=\frac{\sum_{i=1}^{\infty} \int_{0}^{1} \int_{0}^{1} U_{T G}(Y, Z) \tilde{\Psi}_{i}(Y, Z) d Y d Z \bar{F}_{s_{i}}(X)}{\int_{0}^{1} \int_{0}^{1} U_{T G}(Y, Z) d Y d Z}, s=T G, D G, M G, G L, B
$$




$$
F_{A, A V}(X)=\frac{\sum_{i=1}^{\infty} \int_{0}^{1} \int_{0}^{1} U_{T G}(Y, Z) \tilde{\Gamma}_{i}(Y, Z) d Y d Z \bar{F}_{A h_{i}}(X)}{\iint_{0}^{1} U_{0}^{1} U_{T G}(Y, Z) d Y d Z}+F_{A o}
$$

The built-in function NDSolve of the Mathematica system [40] is a suitable computational tool for numerically handling the coupled nonlinear transformed system, eqs.(14), since it provides automatic error controlled solutions and allow for expressing the dependence of the transformed fields along the variable $X$ as continuous interporlation functions.

\subsection{CIEA for Improved Lumped-Differential Formulation}

The Coupled Integral Equations Approach (CIEA) [14-20] aims at reducing the number of independent variables in partial differential equations, via averaging (lumping) operations in the coordinates selected to be eliminated, but partially preserving the local information in these space variables through the corresponding boundary conditions. The use of Hermite formulae to approximate the averaging integrals [21] provides improved formulations when compared to the classical lumped system analysis [20], which is the simplest possible lumped approximation. The partial differential three-dimensional model shown in eqs. (1) is here reformulated using the CIEA technique, yielding a nonlinear coupled mathematical model composed only of first order ODEs that enables simulations with reduced computational costs [14-20].

Following the CIEA procedure, the lumping was applied in the transverse and vertical directions, coordinates $\mathrm{Z}$ and $\mathrm{Y}$, respectively [71]. The approximation $\mathrm{H}_{0,0}$, which corresponds to the trapezoid rule, was used to approximate the average mass flow of the species, while the approximation $\mathrm{H}_{1,1}$, which corresponds to the corrected trapezoid rule, was used to approximate the average species concentrations. In general form, the approximations $\mathrm{H}_{0,0}$ and $\mathrm{H}_{1,1}$ are given by:

$$
\begin{gathered}
H_{0,0} \Rightarrow \int_{0}^{h} f(x) d x \approx \frac{h}{2}(f(0)+f(h)) \\
H_{1,1} \Rightarrow \int_{0}^{h} f(x) d x \approx \frac{h}{2}(f(0)+f(h))+\frac{h^{2}}{12}\left(f^{\prime}(0)+f^{\prime}(h)\right)
\end{gathered}
$$

The dimensionless average concentrations in the transverse direction ( $\mathrm{Z}$ coordinate), $\overline{\mathrm{F}}_{\mathrm{s}}$, and the Z-averaged velocity field, $\bar{U}_{T G}(Y)$, are defined as: 


$$
\begin{gathered}
\bar{F}_{s}(X, Y) \equiv \frac{\int_{0}^{1} U_{T G}(Y, Z) F_{s}(X, Y, Z) d Z}{\int_{0}^{1} U_{T G}(Y, Z) d Z} \\
\bar{U}_{T G}(Y)=\int_{0}^{1} U_{T G}(Y, Z) d Z
\end{gathered}
$$

The species balance equations, eqs. (1), are first averaged in the transverse $\mathrm{Z}$ coordinate, neglecting the axial diffusion term in light of the high values of the Peclet number encountered in this application, as reported in [70]. Next, the resulting equations are averaged in the vertical direction ( $\mathrm{Y}$ coordinate) and a system of first order ordinary differential equations is obtained for the transversally averaged concentrations, given by [71]:

$$
\begin{gathered}
\overline{\bar{U}}_{T G} \frac{d \overline{\bar{F}}_{s}(X)}{d X}=\varsigma \overline{\bar{G}}_{s} \quad \text { where } \mathrm{s}=\mathrm{TG}, \mathrm{DG}, \mathrm{MG}, \mathrm{GL}, \mathrm{B} \\
\overline{\bar{U}}_{T G} \frac{d \overline{\bar{F}}_{A}(X)}{d X}=\xi_{A}\left(3 P^{*} \overline{\bar{F}}_{A}(X)+Q^{*}\right)+\varsigma \overline{\bar{G}}_{A} \\
\overline{\bar{F}}_{T G}(0)=1, \quad \overline{\bar{F}}_{s}(0)=0, \quad \text { where } \mathrm{s}=\mathrm{A}, \mathrm{DG}, \mathrm{MG}, \mathrm{GL}, \mathrm{B}
\end{gathered}
$$

where,

$$
\begin{gathered}
\overline{\bar{U}}_{T G}=\int_{0}^{1} \bar{U}_{T G}(Y) d Y, P^{*}=\frac{-6 A^{*}(1) \overline{\bar{U}}_{T G}}{\bar{U}_{T G}(1)}, Q^{*}=A^{*}(1) F_{A_{0}}\left(4-\left.\frac{1}{\bar{U}_{T G}(1)} \frac{\partial \bar{U}_{T G}}{\partial Y}\right|_{Y=1}\right), \\
A^{*}(Y)=3 \bar{U}_{T G}\left(\frac{1}{\left.\frac{\partial U_{T G}}{\partial Z}\right|_{Z=0}}-\frac{1}{\left.\frac{\partial U_{T G}}{\partial Z}\right|_{Z=1}}\right)
\end{gathered}
$$

The averaged expressions for the non-linear source terms $\overline{\bar{G}}_{\mathrm{s}}$, for the different species in the transesterification reaction, are given by [71]:

$$
\begin{gathered}
\overline{\bar{G}}_{T G}=-k_{1}\left(M \overline{\bar{F}}_{T G} \overline{\bar{F}}_{A}+N \overline{\bar{F}}_{T G}\right)+k_{2} O \overline{\bar{F}}_{D G} \overline{\bar{F}}_{B} \\
\overline{\bar{G}}_{A}=\left(-k_{1} \overline{\bar{F}}_{T G}-k_{3} \overline{\bar{F}}_{D G}-k_{5} \overline{\bar{F}}_{M G}\right) M \overline{\bar{F}}_{A}+\left(-k_{1} \overline{\bar{F}}_{T G}-k_{3} \overline{\bar{F}}_{D G}-k_{5} \overline{\bar{F}}_{M G}\right) N+ \\
+\left(k_{2} \overline{\bar{F}}_{D G}+k_{4} \overline{\bar{F}}_{M G}+k_{6} \overline{\bar{F}}_{G L}\right) O \overline{\bar{F}}_{B} \\
\overline{\bar{G}}_{D G}=\left(k_{1} \overline{\bar{F}}_{T G}-k_{3} \overline{\bar{F}}_{D G}\right) M \overline{\bar{F}}_{A}+\left(k_{1} \overline{\bar{F}}_{T G}-k_{3} \overline{\bar{F}}_{D G}\right) N+\left(-k_{2} \overline{\bar{F}}_{D G}+k_{4} \overline{\bar{F}}_{M G}\right) O \overline{\bar{F}}_{B}
\end{gathered}
$$




$$
\begin{gathered}
\overline{\bar{G}}_{M G}=\left(k_{3} \overline{\bar{F}}_{D G}-k_{5} \overline{\bar{F}}_{M G}\right) M \overline{\bar{F}}_{A}+\left(k_{3} \overline{\bar{F}}_{D G}-k_{5} \overline{\bar{F}}_{M G}\right) N+\left(-k_{4} \overline{\bar{F}}_{M G}+k_{6} \overline{\bar{F}}_{G L}\right) O \overline{\bar{F}}_{B} \\
\overline{\bar{G}}_{G L}=k_{5} M \overline{\bar{F}}_{M G} \overline{\bar{F}}_{A}+k_{5} N \overline{\bar{F}}_{M G}-k_{6} O \overline{\bar{F}}_{G L} \overline{\bar{F}}_{B} \\
\overline{\bar{G}}_{B}=\left(k_{1} \overline{\bar{F}}_{T G}+k_{3} \overline{\bar{F}}_{D G}+k_{5} \overline{\bar{F}}_{M G}\right) M \overline{\bar{F}}_{A}+\left(k_{1} \overline{\bar{F}}_{T G}+k_{3} \overline{\bar{F}}_{D G}+k_{5} \overline{\bar{F}}_{M G}\right) N+ \\
+\left(-k_{2} \overline{\bar{F}}_{D G}-k_{4} \overline{\bar{F}}_{M G}-k_{6} \overline{\bar{F}}_{G L}\right) O \overline{\bar{F}}_{B}
\end{gathered}
$$

where,

$$
\begin{gathered}
M=\frac{-3 \bar{U}_{T G}^{2}\left(B(1)\left(\left.\frac{\partial \bar{U}_{T G}}{\partial Y}\right|_{Y=0}\right)^{2}+6 B(0) \bar{U}_{T G}(1)\left(\left.\frac{\partial \bar{U}_{T G}}{\partial Y}\right|_{Y=1}+2 \bar{U}_{T G}(1)\right)\right)}{\left(\left.\frac{\partial \bar{U}_{T G}}{\partial Y}\right|_{Y=0}\right)^{2} \bar{U}_{T G}(1)\left(\left.\frac{\partial \bar{U}_{T G}}{\partial Y}\right|_{Y=1}-4 \bar{U}_{T G}(1)\right)} \\
N=\frac{-F_{A_{0}} \overline{\bar{U}}_{T G}\left(12 B(0)\left(\bar{U}_{T G}(1)\right)^{2}\left(\left.\frac{\partial \bar{U}_{T G}}{\partial Y}\right|_{Y=1}-2 \bar{U}_{T G}(1)\right)+B(1)\left(\left.\frac{\partial \bar{U}_{T G}}{\partial Y}\right|_{Y=0}\right){ }^{2}\left(\left.\frac{\partial \bar{U}_{T G}}{\partial Y}\right|_{Y=1}+2 \bar{U}_{T G}(1)\right)\right)}{2\left(\left.\frac{\partial \bar{U}_{T G}}{\partial Y}\right|_{Y=0}\right)^{2} \bar{U}_{T G}(1)\left(\left.\frac{\partial \bar{U}_{T G}}{\partial Y}\right|_{Y=1}-4 \bar{U}_{T G}(1)\right)} \\
O=\frac{18 \overline{\bar{U}}_{T G}^{2}\left(B(1)\left(\left.\frac{\partial \bar{U}_{T G}}{\partial Y}\right|_{Y=0}\right)^{2}+B(0)\left(\left.\frac{\partial \bar{U}_{T G}}{\partial Y}\right|_{Y=1}-2 \bar{U}_{T G}(1)\right)^{2}\right)}{\left(\left.\frac{\partial \bar{U}_{T G}}{\partial Y}\right|_{Y=0}\right)^{2}\left(\left.\frac{\partial \bar{U}_{T G}}{\partial Y}\right|_{Y=1}-4 \bar{U}_{T G}(1)\right)^{2}} \\
B^{*}(Y)=18 \bar{U}_{T G}^{2}\left(\frac{1}{\left(\left.\frac{\partial U_{T G}}{\partial Z}\right|_{Z=0}\right)^{2}}-\frac{1}{\left(\left.\frac{\partial U_{T G}}{\partial Z}\right|_{Z=1}\right)^{2}}\right)^{2}
\end{gathered}
$$

\subsection{Inverse Problem Analysis}

Inverse problem analysis is an essential tool in engineering design for the identification of parameters, such as physical properties, and functions, such as equation and boundary condition source terms. Besides, such methodologies have evolved towards experimental planning and optimum design procedures [43-52].

The statistical approach for the solution of inverse problems is briefly reviewed here, following the Markov chain Monte Carlo (MCMC) method [79-81]. The 
MCMC method is used in conjunction with the Coupled Integral Equations Approach for the solution of the inverse problem of estimating the kinetic coefficients in the transesterification reaction, in order to obtain low cost inverse problem solutions, as detailed in [71].

The MCMC method represents a Bayesian approach to parameter estimation. Bayes theorem is given by:

$$
\pi_{\text {posterior }}(\mathbf{P})=\pi(\mathbf{P} \mid \mathbf{Y})=\frac{\pi_{\text {prior }}(\mathbf{P}) \pi(\mathbf{Y} \mid \mathbf{P})}{\pi(\mathbf{Y})}
$$

where $\mathbf{P}$ is the parameter vector, $\mathbf{Y}$ is the vector of measurements, $\pi_{\text {posteriori }}(\mathbf{P})$ is the posterior probability density, $\pi_{\text {prior }}(\mathbf{P})$ is the prior density, $\left.\pi(\mathbf{Y} \mid \mathbf{P})\right)$ is the likelihood function and $\pi(\mathbf{Y})$ is the probability density of the measurements, which represents a normalization constant.

Assuming that the measurement errors are additive, uncorrelated, with Gaussian distribution of zero mean, and known covariance matrix, the likelihood function is given by:

$$
\pi(\mathbf{Y} \mid \mathbf{P})=(2 \pi)^{-I / 2}|\mathbf{W}|^{-1 / 2} \exp \left[-\frac{1}{2}\left[\mathbf{Y}-\mathbf{Y}_{e}(\mathbf{P})\right]^{T} \mathbf{W}^{-1}\left[\mathbf{Y}-\mathbf{Y}_{e}(\mathbf{P})\right]\right]
$$

where $\mathbf{Y}_{\boldsymbol{e}}(\mathbf{P})$ is the solution of the direct problem, obtained with a sample of the parameter vector $\mathbf{P}$ and $\mathbf{W}$ is the covariance matrix of the errors in $\mathbf{Y}$.

It is in general not possible to analytically obtain the posterior probability density, thus the MCMC method is used to draw samples of all possible parameters. One of the most widely used algorithms for implementing the MCMC method is known as the Metropolis-Hasting algorithm, as described in [79].

\subsection{Results and Discussion}

The reagents chosen in the present illustration are soybean oil (triglycerides) and methanol, with sodium hydroxide as catalyst. The hybrid approach here reviewed is then employed to report the direct-inverse problem analysis through the evolution of triglyceride conversion in terms of residence time and reaction temperature, with available data for the chosen reagents [69-71,75], as summarized in Table 3. The Generalized Integral Transform Technique (GITT) was employed to handle both the two- and three dimensional formulations of the complete mass transfer problem, eqs. (1) [67-70]. Both situations of a constant coefficients eigenvalue problem and the alternative of including the velocity profile in the eigenvalue problem formulation have been dealt with [69-70], with relative merits that shall be briefly discussed in what follows. Also, the results obtained through the reduced model obtained with the Coupled Integral Equations Approach were verified comparing the reformulated first order ordinary differential equations system with the three-dimensional model results for micro-reactors with square cross-sections $(200 \times 200 \mu \mathrm{m}$ and $400 \times 400 \mu \mathrm{m})$, and length of $2.33 \mathrm{~cm}$. 
Table 3. Data for the direct-inverse problem analysis of biodiesel synthesis in microreactors [69-71, 75].

\begin{tabular}{|c|c|c|c|}
\hline Parameters & Values & Parameters & Values \\
\hline Alcohol & Methanol & $\mathrm{k}_{1}\left[\mathrm{~mol} /\left(\mathrm{m}^{3} \cdot \mathrm{s}\right)\right]$ & $4.368 \times 10^{-6}$ \\
\hline Oil & Soybean & $\mathrm{k}_{2}\left[\mathrm{~mol} /\left(\mathrm{m}^{3} \cdot \mathrm{s}\right)\right]$ & $9.623 \times 10^{-6}$ \\
\hline $\mathrm{Q}_{\mathrm{TG}} / \mathrm{Q}_{\mathrm{A}}$ & 3.4 & $\mathrm{k}_{3}\left[\mathrm{~mol} /\left(\mathrm{m}^{3} \cdot \mathrm{s}\right)\right]$ & $1.88 \times 10^{-5}$ \\
\hline$\mu_{\mathrm{TG}}[\mathrm{Pa} . \mathrm{s}]$ & $5.825 \times 10^{-2}$ & $\mathrm{k}_{4}\left[\mathrm{~mol} /\left(\mathrm{m}^{3} \cdot \mathrm{s}\right)\right]$ & $1.074 \times 10^{-4}$ \\
\hline$\mu_{\mathrm{A}}[\mathrm{Pa} . \mathrm{s}]$ & $5.47 \times 10^{-4}$ & $\mathrm{k}_{5}\left[\mathrm{~mol} /\left(\mathrm{m}^{3} \cdot \mathrm{s}\right)\right]$ & $2.117 \times 10^{-5}$ \\
\hline $\mathrm{D}_{\mathrm{TG}}\left[\mathrm{m}^{2} / \mathrm{s}\right]$ & $1.58 \times 10^{-9}$ & $\mathrm{k}_{6}\left[\mathrm{~mol} /\left(\mathrm{m}^{3} . \mathrm{s}\right)\right]$ & $9.0 \times 10^{-7}$ \\
\hline$\rho_{\mathrm{TG}}\left[\mathrm{kg} / \mathrm{m}^{3}\right]$ & 885 & Reaction & $\begin{array}{c}\text { Activation energy } \\
{\left[\mathrm{J}^{\left.-\mathrm{mol}^{-1}\right]}\right.}\end{array}$ \\
\hline $\mathrm{D}_{\mathrm{A}}\left[\mathrm{m}^{2} / \mathrm{s}\right]$ & $1.182 \times 10^{-10}$ & $\mathrm{TG} \rightarrow \mathrm{DG}$ & 55.0355 \\
\hline $\begin{array}{c}\mathrm{D}_{\mathrm{DG}}, \mathrm{D}_{\mathrm{MG}}, \mathrm{D}_{\mathrm{GL}}, \\
\mathrm{D}_{\mathrm{B}}\left[\mathrm{m}^{2} / \mathrm{s}\right]\end{array}$ & $1.38 \times 10^{-9}$ & $\mathrm{DG} \rightarrow \mathrm{TG}$ & 41.5833 \\
\hline $\mathrm{C}_{\mathrm{TGo}}\left[\mathrm{mol} / \mathrm{m}^{3}\right]$ & 1014 & $\mathrm{DG} \rightarrow \mathrm{MG}$ & 83.1498 \\
\hline $\mathrm{F}_{\mathrm{Ao}}$ & 4.4 & $\mathrm{MG} \rightarrow \mathrm{DG}$ & 61.2906 \\
\hline \multirow[t]{2}{*}{$\mathrm{R}\left[\mathrm{J} \cdot \mathrm{mol}^{-1} \cdot \mathrm{K}^{-1}\right]$} & 8.314 & $\mathrm{MG} \rightarrow \mathrm{GL}$ & 26.8834 \\
\hline & & $\mathrm{GL} \rightarrow \mathrm{MG}$ & 40.1430 \\
\hline
\end{tabular}

The eigenfunction expansions for the average concentrations of the triglyceride and biodiesel species were first analyzed in terms of convergence rate, taking the temperature $\mathrm{T}=298 \mathrm{~K}$ and residence time $\tau=30 \mathrm{~s}$, for each of the eigenvalue problem choices, as shown in Table 4. The GITT solution obtained with the eigenvalue problem accounting for the velocity profile presents an improved behavior in terms of convergence rate, since it needs around 50 terms to reach up to four fully converged significant digits, while the simpler eigenvalue problem with constant coefficient requires, for the same case, around 100 terms. These results highlight the gain, in terms of convergence rates that can be achieved considering more informative eigenvalue problems, which in the present case is the one that considers the velocity profile in its formulation.

Figure 4 illustrates the evolution with residence time of the dimensionless average concentration of each species at the $400 \times 400$ microns microreactor outlet, as obtained by GITT with increasing truncation orders of the eigenfunction expansions, namely, $\mathrm{NT}=1,5$ and 40 . It can be noticed that to the graph scale the results for fairly low truncation orders, such as NT=5, are already in excellent agreement with the fully converged results with the higher truncation order of NT=40 terms. This observation opened up the possibility of using another reduced model, based on low truncation order approximations of the integral transform solution, as successfully accomplished in [70]. The triglyceride concentration, as expected, reduces with the increase of the residence time, while the other species are formed. Thus, the intermediate species reach a maximum value and then start to decrease as well, leading to the formation of biodiesel. 
Table 4. Convergence of eigenfunction expansions for the average concentrations of triglyceride and biodiesel in a square micro-reactor

$$
\left(L=233 \times 10^{-4} \mathrm{~m}, H=W=100 \times 10^{-6} \mathrm{~m}\right) \text {. }
$$

Eigenvalue problem: constant coefficients

\begin{tabular}{ccccccc}
\hline & \multicolumn{2}{c}{$X=0.3$} & \multicolumn{2}{c}{$X=0.5$} & \multicolumn{2}{c}{$X=0.7$} \\
\hline NT & $C_{T G, A v}$ & $C_{B, A v}$ & $C_{T G, A v}$ & $C_{B, A v}$ & $C_{T G, A v}$ & $C_{B, A v}$ \\
\hline 20 & 0.955411 & 0.049206 & 0.904022 & 0.117023 & 0.844553 & 0.210476 \\
40 & 0.955648 & 0.048923 & 0.904303 & 0.116622 & 0.844850 & 0.209973 \\
60 & 0.955815 & 0.048725 & 0.904513 & 0.116322 & 0.845079 & 0.209585 \\
80 & 0.955802 & 0.048740 & 0.904492 & 0.116351 & 0.845054 & 0.209627 \\
100 & 0.955752 & 0.048799 & 0.904426 & 0.116445 & 0.844981 & 0.209751 \\
\hline
\end{tabular}

Eigenvalue problem: variable coefficients

\begin{tabular}{ccccccc}
\hline & \multicolumn{2}{c}{$X=0.3$} & \multicolumn{2}{c}{$X=0.5$} & \multicolumn{2}{c}{$X=0.7$} \\
\hline NT & $C_{T G, A v}$ & $C_{B, A v}$ & $C_{T G, A v}$ & $C_{B, A v}$ & $C_{T G, A v}$ & $C_{B, A v}$ \\
\hline 10 & 0.955449 & 0.049150 & 0.904122 & 0.116853 & 0.844666 & 0.210240 \\
20 & 0.955705 & 0.048851 & 0.904378 & 0.116504 & 0.844923 & 0.209831 \\
30 & 0.955751 & 0.048798 & 0.904427 & 0.116438 & 0.844977 & 0.209748 \\
40 & 0.955761 & 0.048785 & 0.904438 & 0.116423 & 0.844988 & 0.209729 \\
50 & 0.955766 & 0.048781 & 0.904444 & 0.116415 & 0.844997 & 0.209717 \\
\hline
\end{tabular}

Figures 5.a,b present the triglyceride conversion as a function of residence time for the $200 \times 200 \mu \mathrm{m}$ and the $400 \times 400 \mu \mathrm{m}$ cross-sections, respectively, with $\mathrm{T}=45^{\circ} \mathrm{C}$. The dashed lines are for the reduced model, eqs. (22), solved through the NDSolve algorithm [40]. The dotted lines are for the GITT solution of the three-dimensional model, eqs. (1) [69-70]. One can observe the reasonable agreement of the two solutions, corresponding to the reduced model and to the GITT simulations of the three-dimensional model, especially for smaller and larger residence times. After the reduced model has been verified, it may be used in the estimation of the kinetic constants, with considerable reduction on the computational effort in the analysis of the inverse problem. 

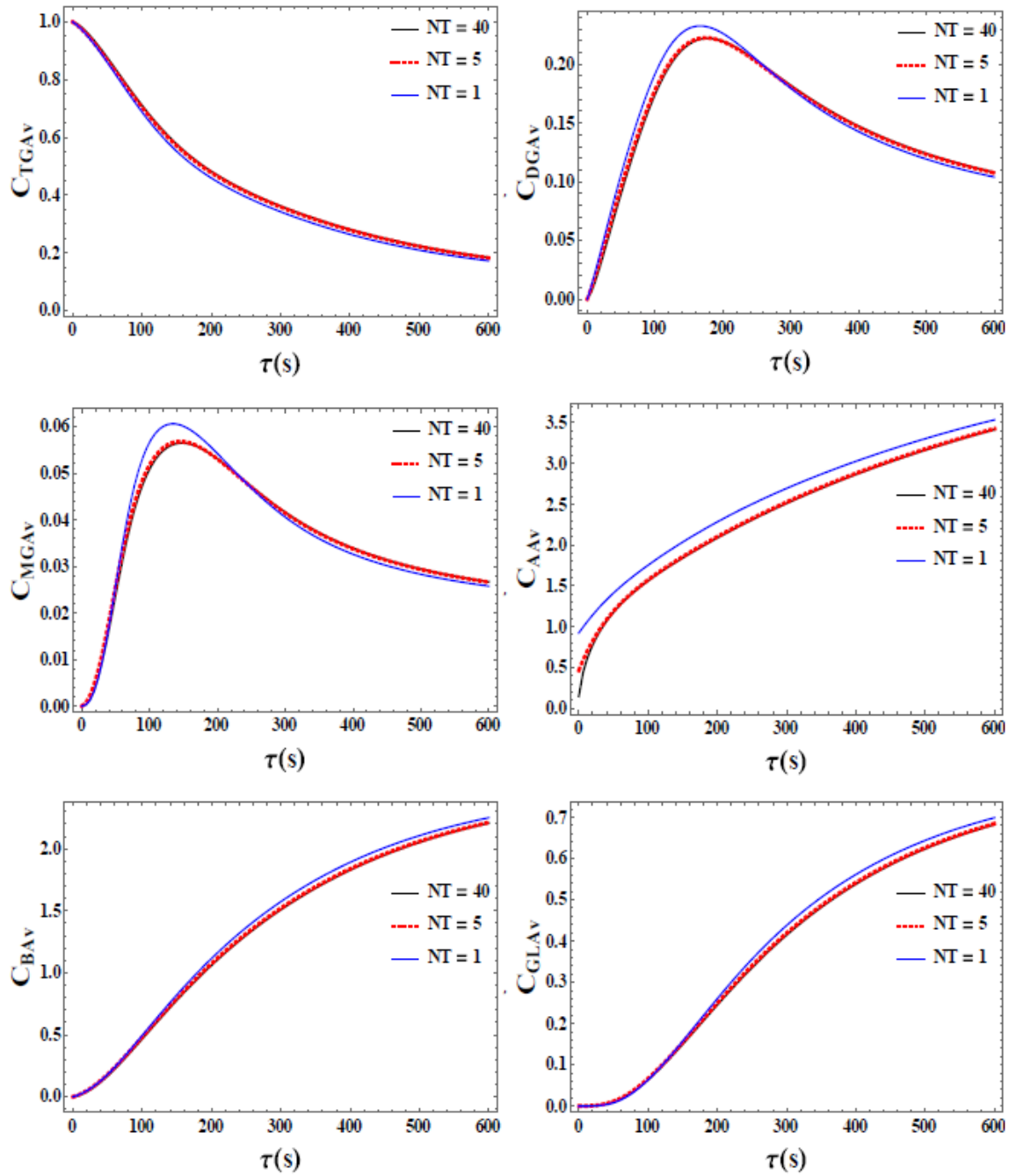

Fig. 4. Average dimensionless concentrations of the different species at the microreactor outlet as computed from the three-dimensional model via GITT for the truncation orders $\mathrm{NT}=1,5$, and 40 . 


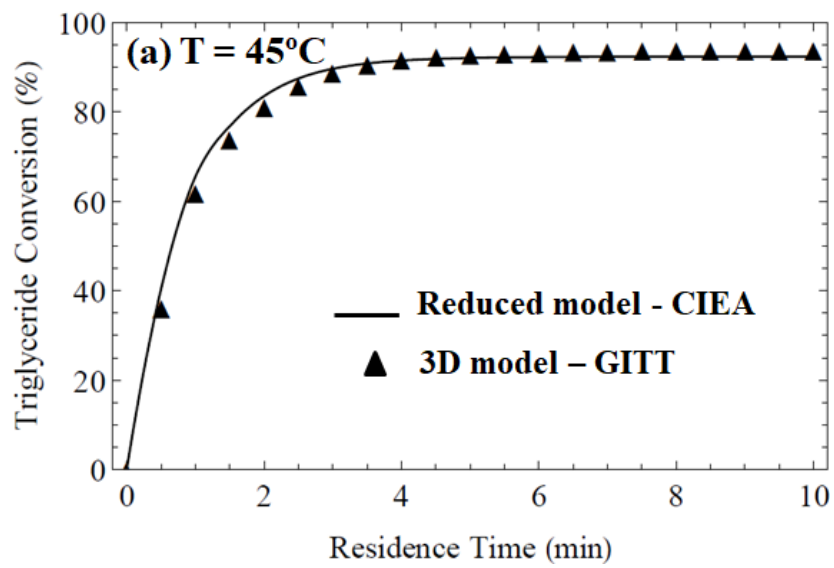

(a) 200x200 microns channel

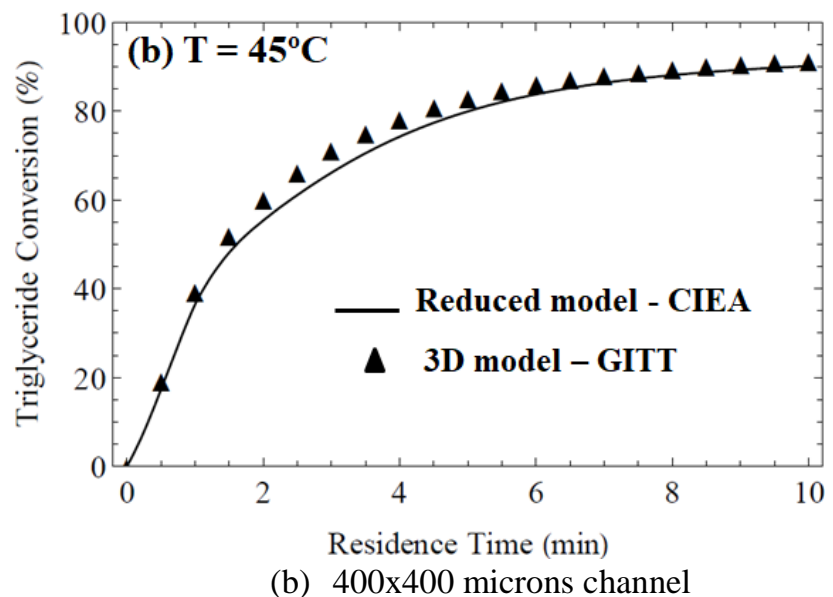

Fig. 5. Comparison of triglycerides conversion in terms of residence time as obtained by the improved lumped-differential formulation (CIEA) and the complete three dimensional model solved by GITT.

In the estimation of the kinetic constants, emphasis is given to the use of the nonlinear coupled first-order ordinary differential system, eqs.(22), using the Coupled Integral Equations Approach (CIEA) for model reduction [71]. The Markov Chain Monte Carlo method (MCMC) is the tool in the inverse problem analysis, using simulated experimental data to estimate the kinetic constants, including low triglyceride conversion rates at shorter residence times and lower temperatures. Simulated data is taken at these less efficient conditions so as to achieve higher concentrations of the intermediate species and provide sensitivity to the parameters to be estimated in the inverse problem solution [71]. 
A few results are provided below to demonstrate the MCMC method. The GITT solution for the fully converged three dimensional model, eqs. (1), is employed to construct this synthetic experimental data, obtained with the input data from the literature provided in Table 5, while maintaining the CIEA reformulation for the direct problem solution in the inverse analysis. For the construction of the vector of simulated experimental measurements, results were obtained at twenty different residence times $(0.5,1.0,1.5,3.0,3.5,4.0,5.5,6.5,8.0,8.5,9.5,10.0,12.0,13.5$, $14.0,14.5,16.0,17.5,18.5$ and $19.0 \mathrm{~min}$ ), for the corresponding volumetric flow rates of the triglyceride species of $0.399,0.199,0.133,0.0660,0.0570,0.0498$, $0.0362,0.0307,0.0249,0.0234,0.0210,0.0199,0.0166,0.0148,0.0142,0.0137$, $0.0125,0.0114,0.0108$ and $0.0105 \mathrm{ml} / \mathrm{h}$, respectively. The simulated experimental results were obtained for four species, triglyceride, diglyceride, monoglyceride, and biodiesel. To capture the effect of uncertainties, experimental standard deviation for the synthetic measurements was taken as $5 \%$ of each concentration maximum value, as computed from the three-dimensional model [71]. Table 5 presents the estimated kinetic constants with their relative deviations from the exact values, now employing the simulated experimental data from the complete three dimensional model and introducing the experimental error. The parameters $\mathrm{k}_{1}$ and $\mathrm{k}_{2}$ lead to the largest deviations (14\% and 13\%, respectively), but overall a good agreement with the exact values is achieved. It should be recalled that the deviations in the present results also incorporate the errors in the simplified reduced model, which provides a marked reduction in the computational cost [71]. For instance, the inverse analysis for 100,000 states in the MCMC algorithm, using the CIEA for the direct problem solution, leads to a CPU time reduction of around 9 times with respect to the GITT solution of the three dimensional model with $\mathrm{N}=5$ terms in the eigenfunction expansions. Nevertheless, the GITT solution is still more accurate than the CIEA solution, even for this reduced truncation order. The concentration profiles at the microreactor outlet (at $\mathrm{X}=1$ ) were then computed with the exact and estimated kinetic constants, for all four species, TG, DG, MG and B, as shown in Figure 6. An overall good agreement of the estimated and exact concentrations was achieved, even though a reduced model has been adopted for the direct problem solution. For the $99 \%$ confidence intervals, as expected, larger bounds are achieved upon including the experimental error.

Table 5 - Estimated kinetic constants and relative errors for the case of simulated data with experimental error

\begin{tabular}{cccc}
\hline $\begin{array}{c}\text { Kinetic } \\
\text { constants }\end{array}$ & $\begin{array}{c}\text { Exact } \\
{\left[\mathrm{mol} / \mathrm{m}^{3} . \mathrm{s}\right]}\end{array}$ & $\begin{array}{c}\text { Estimated } \\
{\left[\mathrm{mol} / \mathrm{m}^{3} . \mathrm{s}\right]}\end{array}$ & $\begin{array}{c}\text { Relative } \\
\text { Error }(\%)\end{array}$ \\
\hline $\mathrm{k}_{1}$ & $4.368 \times 10^{-6}$ & $3.729 \times 10^{-6}$ & 14.62 \\
$\mathrm{k}_{2}$ & $9.623 \times 10^{-6}$ & $8.313 \times 10^{-6}$ & 13.61 \\
$\mathrm{k}_{3}$ & $1.880 \times 10^{-5}$ & $2.038 \times 10^{-5}$ & 8.39 \\
$\mathrm{k}_{4}$ & $1.074 \times 10^{-4}$ & $1.174 \times 10^{-4}$ & 9.28 \\
$\mathrm{k}_{5}$ & $2.117 \times 10^{-5}$ & $1.942 \times 10^{-5}$ & 8.25 \\
$\mathrm{k}_{6}$ & $9.000 \times 10^{-7}$ & $8.507 \times 10^{-7}$ & 5.48 \\
\hline
\end{tabular}


As mentioned above, the kinetic constants are essential parameters to be identified in this reaction-convection-diffusion model. The present hybrid approach is thus able to simultaneously estimate all the unknown parameters in the transport phenomena modeling and design the micro-device to meet specific requirements. In the present illustration, methanol has been selected as the alcohol phase, in accordance with the majority of previous works in biodiesel synthesis. However, the final goal of the research was to develop a microsystem for biodiesel synthesis employing the ethanol route [72]. In fact, after obtaining dedicated experimental results and undertaking the proper estimation of the associated kinetic constants, new promising synthesis results have demonstrated a high convergence rate of $99.9 \%$ of TG in $99.6 \%$ of biodiesel in less than 35 seconds, using a molar ratio of 20:1 (ethanol/soybean oil) and $\mathrm{NaOH}$ as catalyst [72].

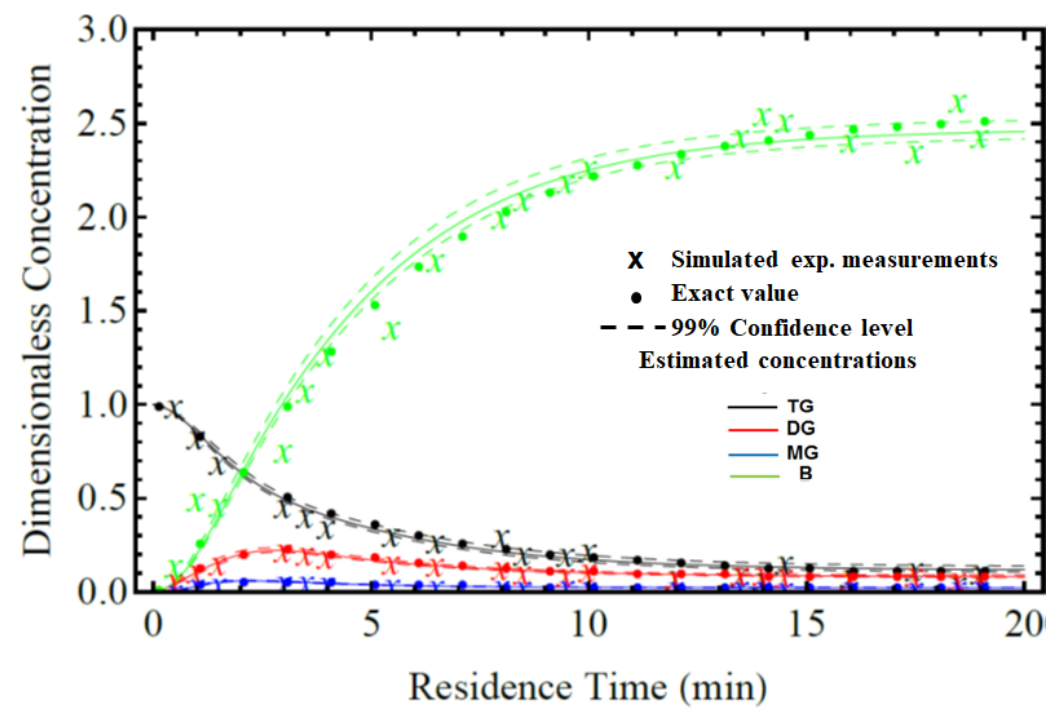

Fig. 6. Estimated and exact concentrations distributions at the micro-reactor exit $(X=1)$ in terms of residence time, including the $99 \%$ confidence level bounds (with experimental error).

\section{CONCLUSIONS}

A hybrid computational-analytical-experimental approach has been reviewed for the analysis of heat and mass transfer phenomena, with emphasis on microscale applications. The approach combines various tools such as a lumped-differential problem reformulation strategy for model reduction, known as the Coupled Integral Equations Approach (CIEA), a hybrid numerical-analytical solution method for partial differential equations based on the Generalized Integral Transform Technique (GITT), and an inverse problem solution implemented in the Bayesian 
inference framework. Following an overall description of the approach and the pertinent literature review, the potential of the proposed hybrid approach is illustrated through an actual application dealing with continuous biodiesel synthesis in microreactors.

\section{ACKNOWLEDGEMENTS}

The authors acknowledge the financial support by CAPES/INMETRO, CNPq and FAPERJ, from Brazil, and the UK Newton Fund. RMC is also grateful to the Leverhulme Trust for the Visiting Professorship (VP1-2017-028) and CNPC acknowledges the post-doctoral fellowship provided by CAPES/Brazil. RMC, CPNC, and PCP acknowledge the hospitality of the Department of Mechanical Engineering, University College London (UCL), UK.

\section{REFERENCES}

1. US NIC (United States National Intelligence Council), Global Trends 2030: Alternative Worlds, US NIC, Washington DC, USA, pp. 137, 2012.

2. United Nations, The Sustainable Development Goals Report 2016, New York, https://unstats.un.org/sdgs/report/2016/The\%20Sustainable\%20Development\%20Goals\%20Report\%202016.pdf, 2016.

3. Ohadi, M.M., K. Choo, S. Dessiatoun, E. Cetegen, Next Generation Microchannel Heat Exchangers, Springer Briefs in Thermal Engineering and Applied Science, Springer-Verlag, 2013.

4. Steinke, M.E., and S.G. Kandlikar, Single-phase Heat Transfer Enhancement Techniques in Micro-channel and Mini-channel Flow, Proc. of the International Conference on Micro-channels and Mini-channels - ASME, Rochester, NY, June 2004.

5. Yener, Y., S. Kakaç, M. Avelino, T. Okutucu, Single-phase Forced Convection in Micro-channels - a State-of-the-art Review, in: S. Kakaç, L.L. Vasiliev, Y. Bayazitoglu, Y. Yener (Eds.), Microscale Heat Transfer - Fundamentals and Applications, NATO ASI Series, Kluwer Academic Publishers, Netherlands, pp.1-24, 2005.

6. Sobhan, C.B., and G.P. Peterson, Microscale and Nanoscale Heat Transfer: Fundamentals and Engineering Applications, CRC Press, Boca Raton, FL, 2008.

7. Kockmann, N., Transport Phenomena in Micro Process Engineering, SpringerVerlag, Berlin Heidelberg, 2008.

8. Cotta, R.M., C.A.A. Mota, C.P. Naveira-Cotta, J.S. Nunes, M.R. Avelino, F.V. Castellóes, J.N.N. Quaresma, Heat Transfer Enhancement in Laminar Forced Convection: Nanofluids. Microchannels. Structured Surfaces, Invited Keynote 
Lecture, ICHMT Digital Library, Proc. of International Symposium on Convective Heat and Mass Transfer in Sustainable Energy - CONV-09, 38p., Yasmine Hammamet, Tunisia, April 2009.

9. Rebay, M., S. Kakaç, and R.M. Cotta, Eds., Microscale and Nanoscale Convective Heat Transfer: Concepts, Analysis, and Applications, CRC Press, Boca Raton, FL, 2016. ISBN: 978-1-498-73630-5

10. Cotta, R.M., D.C. Knupp, and C.P. Naveira-Cotta, Analytical Heat and Fluid Flow in Microchannels and Microsystems, Mechanical Eng. Series, SpringerVerlag, 2016. ISBN: 978-3-319-23311-6

11. Maitra, T., S. Zhang, and M. K. Tiwari, Thermal Transport in Micro and Nanoscale Systems, In: Handbook of Thermal Science and Engineering, F.A. Kulacki et al., Eds., Springer-Verlag, 2017.

12. Cotta, R. M., and H.R.B. Orlande, Hybrid Approaches in Heat and Mass Transfer: A Brazilian Experience with Applications in National Strategic Projects, Heat Transfer Eng., Invited Editorial, V.24, no.4, pp.1-5, 2003.

13. Cotta, R.M., J. Su, A.C. Pontedeiro, and K.M. Lisboa, Computational-Analytical Integral Transforms and Lumped-Differential Formulations: Benchmarks and Applications in Nuclear Technology, Invited Plenary Lecture, Int. Symp. on Turbulence, Heat and Mass Transfer, THMT-ICHMT, Begell House, Rio de Janeiro, July 10th-13 $3^{\text {th }}, 2018$.

14. Aparecido, J.B, and R.M. Cotta, Improved One-Dimensional Fin Solutions, Heat Transf. Eng., V. 11, no.1, pp. 49-59, 1989.

15. Cotta, R.M., M.N. Ozisik, and J. Mennig, Coupled Integral Equation Approach for Phase-Change Problem in Two-Regions Finite Slab, J. of the Franklin Institute, V. 327, no. 2, pp. 225-234, 1990.

16. Cotta, R.M., and M.D. Mikhailov, Heat Conduction: Lumped Analysis, Integral Transforms, Symbolic Computation, Wiley Interscience, New York, USA, 1997.

17. Correa, E.J., and R.M. Cotta, Enhanced Lumped-Differential Formulations of Diffusion Problems, Appl. Math. Modeling, V.22, pp.137-152, 1998.

18. Cotta, R.M., Improved Lumped-Differential Formulations in Heat Transfer, Invited Chapter, Volume 2 - Modelling of Engineering Heat Transfer Phenomena, Chap.10, Eds. B. Sunden and M. Faghri, Heat Transfer Series, Computational Mechanics Publ., UK, pp.293-325, 1998.

19. Alves, L.S.B., L.A. Sphaier, and R.M. Cotta, Error Analysis of Mixed LumpedDifferential Formulations in Diffusion Problems, Hybrid Meth. Eng., V.2, no.4, pp.409-435, 2000.

20. Sphaier, L.A., J. Su, and R.M. Cotta, Macroscopic Heat Conduction Formulation, In: Handbook of Thermal Science and Engineering, F.A. Kulacki et al., Eds., Springer, 2017.

21. Hermite, M.C., Sur la Formule d'Interpolation de Lagrange, J Crelle, V. 84, 1878.

22. Hirata, S.C., B. Goyeau, D. Gobin, M. Carr, and R.M. Cotta, Linear Stability of Natural Convection in Superposed Fluid and Porous Layers: Influence of the Interfacial Modelling, Int. J. Heat \& Mass Transfer, V. 50, pp. 1356-1367, 2007. 
23. Hirata, S.C., B. Goyeau, D. Gobin, M. Chandesris, and D. Jamet, Stability of Natural Convection in Superposed Fluid and Porous Layers: Equivalence of the One- and Two-domain Approaches, Int. J. Heat \& Mass Transfer, V. 52, pp. 533-536, 2009.

24. Knupp, D.C., C.P. Naveira-Cotta, and R.M. Cotta, Theoretical Analysis of Conjugated Heat Transfer with a Single Domain Formulation and Integral Transforms, Int. Comm. Heat \& Mass Transfer, V.39, no.3, pp.355-362, 2012.

25. Knupp, D.C., R.M. Cotta, and C.P. Naveira-Cotta, Fluid Flow and Conjugated Heat Transfer in Arbitrarily Shaped Channels via Single Domain Formulation and Integral Transforms, Int. J. Heat \& Mass Transfer, V. 82, pp.479-489, 2015.

26. Minkowycz, W.J., E.M. Sparrow, and J.Y. Murthy, Eds., Handbook of Numerical Heat Transfer, $2^{\text {nd }}$ edition, Chapter 16, John Wiley, New York, 2006.

27. Luikov, A.V., Analytical Heat Diffusion Theory, Academic Press, New York, 1968 .

28. Ozisik, M.N., Heat Conduction, John Wiley, New York, 1980.

29. Mikhailov, M.D., and M.N. Ozisik, Unified Analysis and Solution of Heat and Mass Diffusion, John Wiley, 1984; also, Dover Publications, 1993.

30. Cotta, R.M., Diffusion in Media with Prescribed Moving Boundaries: Application to Metals Oxidation at High Temperatures, Proc. of the II Latin American Congress of Heat \& Mass Transfer, V. 1, pp. 502-513, São Paulo, Brasil, May 1986.

31. Cotta, R.M., and M.N. Ozisik, Laminar Forced Convection in Ducts with Periodic Variation of Inlet Temperature, Int. J. Heat Mass \& Transfer, V. 29, no. 10, pp.1495-1501, 1986.

32. Cotta, R.M., Hybrid Numerical-Analytical Approach to Nonlinear Diffusion Problems, Num. Heat Transfer, Part B, V. 127, pp.217-226, 1990.

33. Cotta, R.M., Integral Transforms in Computational Heat and Fluid Flow, CRC Press, Boca Raton, FL, USA, 1993.

34. Cotta, R.M., Benchmark Results in Computational Heat and Fluid Flow: -The Integral Transform Method, Int. J. Heat \& Mass Transfer, Invited Paper, V.37, pp.381-394, 1994.

35. Cotta, R.M., The Integral Transform Method in Thermal \& Fluids Sciences \& Engineering, Begell House, New York, USA, 1998.

36. Cotta, R.M., and M.D. Mikhailov, Hybrid Methods and Symbolic Computations, Handbook of Numerical Heat Transfer, 2nd ed., Chap. 16, W.J. Minkowycz, E.M. Sparrow, and J.Y. Murthy, Eds., John Wiley, New York, 2006.

37. Cotta, R.M., D.C. Knupp, C.P. Naveira-Cotta, Analytical Heat and Fluid Flow in Microchannels and Microsystems, Mechanical Eng. Series, Springer, New York, 2016.

38. Cotta, R.M., D.C. Knupp, and J.N.N. Quaresma, Analytical Methods in Heat Transfer, In: Handbook of Thermal Science and Engineering, F.A. Kulacki et al., Eds., Springer, 2017. 
39. Cotta, R.M., C.P. Naveira-Cotta, D.C. Knupp, J.L.Z. Zotin, P.C. Pontes, and A.P. Almeida, Recent Advances in Computational-Analytical Integral Transforms for Convection-Diffusion Problems, Heat \& Mass Transfer, Invited Paper, V.54, pp.2475-2496, 2018.

40. Wolfram, S., Wolfram Mathematica, version 11, Wolfram Research Inc., Champaign, IL, USA, 2017.

41. Cotta, R.M., D.C. Knupp, C. P. Naveira-Cotta, L.A. Sphaier, and J.N.N. Quaresma, Unified Integral Transforms Algorithm for Solving Multidimensional Nonlinear Convection-Diffusion Problems, Num. Heat Transfer, Part A-Applications, V.63, pp.1-27, 2013.

42. Cotta, R.M., D.C. Knupp, C.P. Naveira-Cotta, L.A. Sphaier, and J.N.N. Quaresma, The Unified Integral Transforms (UNIT) Algorithm with Total and Partial Transformation, Computational Thermal Sciences, V.6, no.6, pp.507-524, 2014.

43. Ozisik, M.N., and H.R.B. Orlande, Inverse Heat Transfer: Fundamentals and Applications, Taylor and Francis, New York, 2000.

44. Orlande, H.R.B., O. Fudym, D. Maillet, R.M. Cotta, Thermal Measurements and Inverse Techniques, CRC Press, Boca Raton, FL, USA, 2011.

45. Naveira-Cotta, C.P., H.R.B. Orlande, and R.M. Cotta, Inverse Analysis of Forced Convection in Microchannels with Slip Flow via Integral Transforms and Bayesian Inference, Int. J. Thermal Sciences, V.49, pp.879-888, 2010.

46. Naveira-Cotta, C.P., H.R.B. Orlande, R.M. Cotta, Integral Transforms and Bayesian Inference in the Identification of Variable Thermal Conductivity in Two-Phase Dispersed Systems, Numerical Heat Transfer, Part B- Fundamentals, V. 57, pp.173-202, 2010.

47. Naveira-Cotta, C.P., R.M. Cotta, and H.R.B. Orlande, Inverse Analysis with Integral Transformed Temperature Fields for Identification of Thermophysical Properties Functions in Heterogeneous Media, Int. J. Heat \& Mass Transfer, V.54, no.7-8, pp.1506-1519, 2011.

48. Naveira-Cotta, C.P., R.M. Cotta, and H.R.B. Orlande, Estimation of Space Variable Thermophysical Properties, In: Thermal Measurements and Inverse Techniques, H.R.B. Orlande, O. Fudym, D. Maillet, and R.M. Cotta, Eds., Chap.20, pp.675-707, CRC Press, Boca Raton, FL, USA, 2011.

49. Knupp, D.C., C.P. Naveira-Cotta, J.V.C. Ayres, H.R.B. Orlande, and R.M. Cotta, Space-Variable Thermophysical Properties Identification in Nanocomposites via Integral Transforms, Bayesian Inference and Infrared Thermography, Inverse Problems in Science \& Eng., V.20, no.5, pp.609-637, 2012.

50. Knupp, D.C., C.P. Naveira-Cotta, J.V.C. Ayres, R.M. Cotta, and H.R.B. Orlande, Theoretical-Experimental Analysis of Heat Transfer in Nonhomogeneous Solids Via Improved Lumped Formulation. Integral Transforms and Infrared Thermography, Int. J. Thermal Sciences, V.62, pp.71-84, 2012.

51. Knupp, D.C., C.P. Naveira-Cotta, H.M. Fonseca, H.R.B. Orlande, R.M. Cotta, and O. Fudym, Thermal Characterization of Non-homogeneous Media, In: Modelling and Simulation of Diffusive Processes, Springer Series: Simulation Foundations, Methods and Applications, S.K. Basu and N. Kumar, Eds., Chapter 6, pp.119-139, 2014. 
52. Abreu, L.A.S., H.R.B. Orlande, M.J. Colaço, J. Kaipio, V. Kolehmainen, and R.M. Cotta, Detection of Contact Failures with the Markov Chain Monte Carlo Method by Using Integral Transformed Measurements, Int. J. Thermal Sciences, V.132, pp.486-497, 2018.

53. Hetsroni, G., A. Mosyak, E. Pogrebnyak, and L.P. Yarin, Heat Transfer in Microchannels: Comparison of Experiments with Theory and Numerical Results, Int. J. Heat \& Mass Transfer, V. 48, pp. 5580-5601, 2005.

54. Naveira-Cotta, C.P., H.R.B. Orlande, R.M. Cotta, and J.S. Nunes, Integral Transforms, Bayesian Inference, and Infrared Thermography in the Simultaneous Identification of Variable Thermal Conductivity and Diffusivity in Heterogeneous Media, 14th Int. Heat Transfer Conf., Paper no. IHTC14-22511, V.3, pp. 341-350, Washington, DC, USA, August 2010.

55. Knupp, D.C., C.P. Naveira-Cotta, J.V.C. Ayres, H.R.B. Orlande, and R.M. Cotta, Experimental-Theoretical Analysis in a Transient Heat Conduction Setup via Infrared Thermography and Unified Integral Transforms, Int. Review Chemical Eng., IRECHE, V.2, no.6, pp.736-747, 2010.

56. Knupp, D.C., C.P. Naveira-Cotta, H.R.B. Orlande, and R.M. Cotta, Experimental Identification of Thermophysical Properties in Heterogeneous Materials with Integral Transformation of Temperature Measurements from Infrared Thermography, Exp. Heat Transfer, V.26, pp. 1-25, 2013.

57. Abreu, L.A., H.R.B. Orlande, J. Kaipio, V. Kolehmainen, R.M. Cotta, and J.N.N. Quaresma, Identification of Contact Failures in Multi-Layered Composites with the Markov Chain Monte Carlo Method, ASME J. Heat Transfer, V.136, no.10, pp.101302-311, 2014.

58. Costa Junior, J.M., C.P. Naveira-Cotta, and O. Fudym, Image Processing of Temperature Fields from Infrared Termography of Micro-Mixers with Polymeric Substrates, Thermal Engineering (RETERM), V.13, no.1, pp.71-79, 2014.

59. Knupp, D.C., C.P. Naveira-Cotta, A. Renfer, M.K. Tiwari, R.M. Cotta, and D. Poulikakos, Analysis of Conjugated Heat Transfer in Micro-Heat Exchangers via Integral Transforms and Non-Intrusive Optical Techniques, Int. J. Num. Meth. Heat \& Fluid Flow, V.25, no.6, pp.1444-1462, 2015.

60. Renfer, A., M.K. Tiwari, T. Brunschwiler, B. Michel, and D. Poulikakos, Experimental Investigation into Vortex Structure and Pressure Drop Across Microcavities in 3D Integrated Electronics, Experiments in Fluids, V.51, pp. 731$741,2011$.

61. Renfer, A., M.K. Tiwari, R. Tiwari, F. Alfieri, T. Brunschwiler, B. Michel, and D. Poulikakos, Microvortex-enhanced Heat Transfer in 3D-integrated Liquid Cooling of Electronic Chip Stacks, Int. Journal Heat \& Mass Transfer, V.65, pp.33-43, 2013.

62. Cagney, N., and S. Balabani, Streamwise Vortex-Induced Vibrations of Cylinders with One and Two Degrees-of-Freedom, J. Fluid Mech., V.758. pp. 702$727,2014$.

63. Ma, S., J.M. Sherwood, W.T.S. Huck, and S. Balabani, On the Flow Topology Inside Droplets Moving in Rectangular Microchannels, Lab Chip, V.14, pp.3611-3620, 2014. 
64. Sharma, C.S., M.K. Tiwari, S. Zimmermann, T. Brunschwiler, G. Schlottig, B. Michel, and D. Poulikakos, Energy Efficient Hotspot-targeted Embedded Liquid Cooling of Electronics, Applied Energy, V.138, pp.414-422, 2015.

65. Zhang, S., M.K. Tiwari, S. Balabani, C.P. Naveira-Cotta, and R. M. Cotta, Residence Time Control in Micromixers Based on Vortex Shedding, 9th World Conference on Experimental Heat Transfer, Fluid Mechanics and Thermodynamics, Iguaçu Falls, Brazil, 12-15 June 2017.

66. Costa Junior, J.M., C.P. Naveira-Cotta, J.S. Nunes, and C.P. Tostado, Design, Fabrication and Characterization of Micro-Reactor for Biodiesel Synthesis, Heat Pipe Science and Technology, V.6, nos.3-4, pp.135-153, 2015.

67. Pontes, P.C., K. Chen, C.P. Naveira-Cotta, J.M. Costa Junior, C.P. Tostado, and J.N.N. Quaresma, Mass Transfer Simulation of Biodiesel Synthesis in Microreactors, Computers \& Chemical Eng., V.93, pp.36-51, 2016.

68. Pontes, P.C., and C.P. Naveira-Cotta, Inverse Problem Analysis for Identification of Reaction Kinetics Constants in Microreactors for Biodiesel Synthesis, Journal of Physics: Conference Series, V.745, 2016.

69. Pontes, P.C., C.P. Naveira-Cotta, E.N. Macêdo, and J.N.N. Quaresma, Integral Transforms Analysis of Three-Dimensional Mass Transfer in The Transesterification Process in Micro-Reactors, In: Proceedings of the International Symposium on Advances in Computational Heat Transfer - ICHMT, Piscataway, USA, May 2015.

70. Pontes, P.C., C.P. Naveira-Cotta, and J.N.N. Quaresma, Three-dimensional Reaction-convection-diffusion Analysis with Temperature Influence for Biodiesel Synthesis in Micro-reactors, Int. J. Thermal Sciences, V.118, pp.104-122, 2017.

71. Costa Junior, J.M., and C.P. Naveira-Cotta, Estimation of Kinetic Constants in Micro-Reactors for Biodiesel Synthesis: Bayesian Inference with Reduced Mass Transfer Model, Chem. Eng. Research \& Design, V.141, pp.550-565, 2019.

72. Costa Junior, J.M., Theoretical-Experimental Analysis of Microreactors for Biodiesel Synthesis with Rejected Heat Recovery, DSc Thesis, Mechanical Eng. Dept., PEM/COPPE, Federal University of Rio de Janeiro, UFRJ, 2017.

73. de Souza, I.F., D. Guerrieri, C.P. Naveira-Cotta, and M.K. Tiwari, Experimental-Theoretical Analysis of a Micro Heat Exchanger for Waste Heat Recovery from HCPV Systems, ASME Journal of Thermal Sciences and Eng. Applications, doi:10.1115/1.4041439, published on line Sept. $3^{\text {rd }}, 2018$.

74. Schwarz, S., E.S. Borovinskaya, and W. Reschetilowski, Base Catalyzed Ethanolysis of Soybean Oil in Micro-reactors: Experiments and Kinetic Modeling, Chemical Eng. Science, V. 104, pp.610-618, 2013.

75. Al-Dhubabian, A.A., Production of Biodiesel from Soybean Oil in a Micro Scale Reactor, M.Sc. thesis, Oregon State University, Corvallis, OR, USA, 2005.

76. Billo, R.E., C.R. Oliver, R. Charoenwat, B.H. Dennis, P.A. Wilson, J. W. Priest, H. Beardsley, A Cellular Manufacturing Process for a Full-Scale Biodiesel Microreactor, J. Manufacturing Systems, V. 1, pp.409-416, 2015. 
77. Santana, H.S., D.S. Tortola, E.M. Reis, J.L. Silva Jr., and O.P. Taranto, Transesterification Reaction of Sunflower Oil and Ethanol for Biodiesel Synthesis in Microchannel Reactor: Experimental and Simulation Studies, Chemical Eng. Journal, V. 302, pp. 752-762, 2016.

78. Tiwari, A., V.M. Rajesh, S. Yadav, Biodiesel Production in Micro-reactors: A Review", Energy for Sustainable Development, V.43, pp. 143-161, 2018.

79. Kaipio, J., and E. Somersalo, Statistical and Computational Inverse Problems, Applied Mathematical Sciences, V. 160, Springer-Verlag, 2004.

80. Gamerman, D., and H.F. Lopes, Markov Chain Monte Carlo: Stochastic Simulation for Bayesian Inference, 2nd ed., Chapman \& Hall/CRC, Boca Raton, FL, USA, 2006.

81. Orlande, H.R.B., Inverse Problems in Heat Transfer: New Trends on Solution Methodologies and Applications, ASME J. Heat Transfer, V.134, pp.031011, 2012. 